\title{
Sneutrino dark matter via pseudoscalar $X$-funnel meets inverse seesaw
}

\author{
Jung Chang, ${ }^{a, b}$ Kingman Cheung, ${ }^{b, c, d}$ Hiroyuki Ishida, ${ }^{b}$ Chih-Ting Lu, ${ }^{c}$ \\ Martin Spinrath ${ }^{b}$ and Yue-Lin Sming Tsai ${ }^{e}$ \\ ${ }^{a}$ Department of Physics, Chonnam National University, \\ 300 Yongbong-dong, Buk-gu, Gwangju, 500-757, Republic of Korea \\ ${ }^{b}$ Physics Division, National Center for Theoretical Sciences, \\ Hsinchu 30013, Taiwan \\ ${ }^{c}$ Department of Physics, National Tsing Hua University, \\ Hsinchu 30013, Taiwan \\ ${ }^{d}$ Division of Quantum Phases 6 Devices, School of Physics, Konkuk University, \\ Seoul 143-701, Republic of Korea \\ ${ }^{e}$ Institute of Physics, Academia Sinica, \\ Nangang, Taipei 11529, Taiwan \\ E-mail: lovejesus99wwjd@gmail.com, cheung@phys.nthu.edu.tw, \\ hiroyuki403@cts.nthu.edu.tw, timluyu@hotmail.com, \\ martin.spinrath@cts.nthu.edu.tw, smingtsai@gate.sinica.edu.tw
}

ABSTRACT: In this paper we study sneutrino dark matter in a recently proposed supersymmetric electroweak-scale inverse seesaw model, in which the majority of the sneutrino dark matter particle is a mixture of the right-handed sneutrino $\tilde{N}^{c}$ and the singlet field $\tilde{S}$. The scalar field $X$ responsible for the generation of neutrino masses can simultaneously play a crucial role for sneutrino annihilation in the early Universe via the pseudoscalar mediator $A_{X}$ into neutrinos. We focus here on the dominant annihilation channels and provide all the formulas together with analytic estimates in order to identify the relevant parameters. Furthermore, we show that the direct detection scattering cross section is many orders of magnitude below the current limits, and estimate the indirect detection annihilation rate, which is only a few orders of magnitude below the current limits.

KEYWORDS: Supersymmetry Phenomenology

ARXIV EPRINT: 1806.04468 


\section{Contents}

1 Introduction 1

2 Stability of the lightest supersymmetric particle $\quad 2$

3 Masses of sneutrinos, neutralinos, and Higgs fields 4

3.1 Sneutrinos 5

$\begin{array}{lll}3.2 & \text { Neutralinos } & 7\end{array}$

$\begin{array}{lll}3.3 & \text { Higgs bosons } & 8\end{array}$

4 Sneutrino dark matter $\quad 11$

$\begin{array}{lll}4.1 & \text { Numerical calculation of the particle spectra } & 11\end{array}$

4.2 (Co-)annihilation cross sections and relic density 14

4.2.1 Scenario A: sneutrino co-annihilation via the $A_{X}$-funnel $\quad 15$

4.2.2 Scenario H: sneutrino annihilation via the $H_{X}$-funnel 19

4.3 Dark matter direct and indirect detection $\quad 21$

$\begin{array}{lll}\text { 4.3.1 Dark matter direct detection } & 21\end{array}$

4.3.2 Dark matter indirect detection 22

5 Summary and conclusions $\quad 24$

\section{Introduction}

There are many well-established experimental hints for the existence of dark matter (DM) in the Universe, for instance, gravitational effects on visible matter, e.g., galactic rotational velocities, structure formation, etc. However, the nature of DM is still unknown [1]. So far all the terrestrial experiments looking for a direct scattering signal with the DM particles fail to see anything. A large portion of the mass range and interaction strength of the so-called weakly-interacting massive particles (WIMP) are ruled out. The focus shifts now to lighter mass ranges (sub-GeV) or other types of DM like axions.

Another undeniable evidence of physics beyond the Standard Model (SM) is neutrino masses and oscillations [2,3]. An interesting possibility is that DM may be deeply related to the generation of neutrino masses, for which we study an example here. In a previous work, we have proposed a supersymmetric inverse seesaw (ISS) model [4], in which the neutrino mass scale is related to the supersymmetry (SUSY) breaking scale. This is in contrast to many other ISS models where the right-handed neutrino masses are forbidden and the smallness of the lepton number violating masses are put in by hand. The model that we proposed has a built-in $Z_{3} \times Z_{2}$ symmetry, in which the $Z_{2}$ factor is identical to the matter parity, which guarantees the stability of the lightest supersymmetric particle 
(LSP) in our model and all $R$-parity violating operators are automatically forbidden as we will explain in more detail later. Thus, the LSP can be a DM candidate. In this work, we work out explicitly the link between the neutrino mass generation and the DM particle in our model.

In the conventional constrained Minimal Supersymmetric Standard Model (MSSM), the most popular LSP or DM candidate is the lightest neutralino, and the parameter space for viable DM fall into (i) co-annihilation region, (ii) focus-point region, or (iii) $A$-funnel region. In all these three regions, fairly strong annihilation rates are needed for the LSP, so that it would not be overproduced in the early Universe. In particular, the $A$-funnel region is where the pseudoscalar mass is roughly twice of the DM mass such that the annihilation occurs very close to the resonance. The DM candidate in our model shares a similar spirit as the $A$-funnel in the MSSM as we will elaborate in subsequent sections.

In the supersymmetric ISS model that we proposed [4], there are extra superfields $\hat{N}^{c}, \hat{S}$, and $\hat{X}$ in addition to the conventional superfields in the MSSM. Here $\hat{N}^{c}$ and $\hat{S}$ are lepton-like particles and their fermionic components mix with the SM neutrinos after symmetry breaking. The neutrino mass matrix exhibits an ISS-like structure and thus, light neutrino masses can be generated with TeV-scale mass parameters of the model. The vacuum expectation value (vev) of $X$ for that matter generates the lepton number breaking components of the Majorana neutrino mass matrix. In this model the lightest sneutrino can be the DM candidate, which is a scalar particle in contrast to the usual neutralino DM. The purely left-handed sneutrino DM in the MSSM faces two problems because of its sizeable couplings with the $Z$ boson: i) the relic density is too small [5, 6] and/or ii) a large direct detection cross section [7]. Some remedies exist in the literature where the sneutrino is a linear combination of left-handed and right-handed components, e.g., [812]. There are even models where the lightest sneutrino is purely right-handed and thus a viable DM candidate [13-21]. In our setup the lightest sneutrino is mostly a mixture of scalar components of $\hat{N}$ and $\hat{S}$ but it contains a tiny fraction of left-handed sneutrinos as well. We assume that the sneutrinos are produced thermally in the early Universe and annihilate via the $H_{X^{-}}$or $A_{X}$-funnel, where $H_{X}\left(A_{X}\right)$ is the real (pseudo)scalar component of the complex $X$-scalar. We will show that only the $A_{X}$-funnel is sufficiently efficient to get the right relic density.

The organization of the work is as follows. In the next section, we discuss the stability of the lightest sneutrino as the DM using symmetry arguments and compare our model to other supersymmetric models in the literature. In section 3, we present formulas for the mass spectra for sneutrinos, neutralinos, and Higgs bosons and in section 4, we calculate the relic density and discuss estimates for direct and indirect detection experiments. Finally, we summarize and conclude in section 5 .

\section{$2 \quad$ Stability of the lightest supersymmetric particle}

The stability of the LSP is in many cases guaranteed by a (discrete) symmetry. In the MSSM, this is usually either $R$-parity, $P_{R}=(-1)^{3(B-L)+2 s}$ [22], or matter parity, $P_{M}=$ 


\begin{tabular}{|lcccccccccc|}
\hline Superfield & $\hat{Q}_{i}$ & $\hat{U}_{i}^{c}$ & $\hat{E}_{i}^{c}$ & $\hat{L}_{i}$ & $\hat{D}_{i}^{c}$ & $\hat{H}_{u}$ & $\hat{H}_{d}$ & $\hat{N}_{\alpha}^{c}$ & $\hat{S}_{\alpha}$ & $\hat{X}$ \\
\hline$Z_{3}$ charge $\left(q_{3}\right)$ & 1 & 1 & 1 & 0 & 0 & 1 & 2 & 2 & 1 & 1 \\
$Z_{2}$ charge $\left(q_{2}\right)$ & 1 & 1 & 1 & 1 & 1 & 0 & 0 & 1 & 1 & 0 \\
\hline
\end{tabular}

Table 1. Superfield content of the model and charge assignment under the additional discrete $Z_{6}=Z_{3} \times Z_{2}$ symmetry. The new superfields compared to the MSSM, $\hat{N}^{c}, \hat{S}$ and $\hat{X}$, are singlets under the Standard Model gauge group. The indices $i=1,2,3$ and $\alpha=1,2$ are generation indices.

$(-1)^{3(B-L)}[23-26]$. Here $B$ stands for baryon number, $L$ for lepton number, and $s$ for the spin of the particle.

In our model [4] we cannot use any of the two. Lepton number is explicitly broken by the $\hat{X}^{3}$ term in the superpotential. Strangely, although we cannot define matter or $R$-parity using the above definitions, the $R$-parity violating operators of the MSSM are forbidden. This is not an accident as we elucidate now. To understand the symmetries in our model better we can rewrite the $Z_{6}$ symmetry of the original model into an isomorphic $Z_{3} \times Z_{2}$ symmetry, see table 1 .

The $Z_{2}$ factor is identical to matter parity if we restrict ourselves to the MSSM fields. Higgs fields are even under the $Z_{2}$ and matter fields odd. This pattern is still true if we go to the full model. The right-handed neutrinos, $\hat{N}$, and the lepton-like singlets, $\hat{S}$, are odd as they should be since their fermionic components mix with the MSSM neutrinos. The scalar component of $\hat{X}$, which is even under the $Z_{2}$ receives a vev and hence behaves like the Higgs doublets. The $Z_{2}$ symmetry is hence nothing else than a straight-forward extension of matter parity to our model. We just cannot refer in its definition explicitly to any accidental symmetry of the SM. It is also important to note that the $Z_{2}$ remains unbroken after symmetry breaking.

To understand the properties of DM it is convenient to include spin. Now $R$ - and matter parity are known to be equivalent since the product of $(-1)^{2 s}$ for the particles involved in any interaction vertex in a theory that conserves angular momentum is always equal to +1 . This is as well true in our model so that we arrive at what we call DM parity

$$
P_{\mathrm{DM}}=(-1)^{q_{2}+2 s},
$$

where $q_{2}$ is the charge of the corresponding field under the $Z_{2}$ of table 1 . Sneutrinos with $q_{2}=1$ and $2 s=0$ and neutralinos with $q_{2}=0$ and $2 s=1$ are odd under DM parity, $P_{\mathrm{DM}}=-1$. Hence, these two particles are potential DM candidates in our model. ${ }^{1}$

We want to comment here briefly on other models in the literature. Of course, there is a mountain of papers on (SUSY) DM models and it is clearly beyond the scope of our paper to attempt to give a complete review on that. Therefore, we will only comment on supersymmetric inverse seesaw models which discuss DM as well. Most models which we found [11, 27-31] simply extend (implicitly) $R$-parity treating the additional lepton-like fields like MSSM matter fields without any discussion on how this relates explicitly to the

\footnotetext{
${ }^{1}$ The gravitino with $q_{2}=0$ and $2 s=3$ is a potential DM candidate as well, which we nevertheless do not discuss here any further.
} 
lepton number. This can be done without any obvious harm since a neutrino Majorana mass term breaks lepton number by two units and hence does not break $R$-parity explicitly. In their works, sneutrino DM annihilates either via the resonance of a $s$-channel Higgs mediator or through large annihilation into $W W, Z Z$, and $h h$. In ref. [32] the authors introduced a field, $\hat{S}$, whose vev breaks lepton number by two units similar to our $\hat{X}$ field. However, they did not specify any UV completion or symmetries and instead referred to some previous works. In refs. $[33,34]$ they sketched a path towards a UV completion introducing a non-renormalisable operator and a symmetry breaking field $\hat{S}$. They also discussed some tentative UV completions but did not fully specify the symmetries, field content and symmetry breaking potentials. In their approach, the sneutrino can be the asymmetric DM and is favored to be near the electroweak scale.

In refs. [19-21, 35-38] the authors were more explicit (or refer to previous explicit works) and broke a gauged $\mathrm{U}(1)_{B-L}$ symmetry spontaneously. In $[36,37$ to be more precise they started with a left-right symmetric setup inspired by a grand unified theory which contains a $\mathrm{U}(1)_{B-L}$ factor in the breaking chain. The $R$-parity in this cases usually survives as a discrete subgroup ensuring DM stability. Nevertheless, in ref. [37] $R$-parity was broken such that DM can decay. In refs. $[39,40]$ they introduced an additional $\mathrm{U}(1)_{B-L} \times$ $\mathrm{U}(1)_{R}$ symmetry and assumed matter parity on top to guarantee the stability of DM after symmetry breaking and avoiding additional unwanted terms.

In this work, we will focus on thermally produced sneutrino DM which co-annihilates through what we dubbed the $A_{X}$-funnel. In our setup the complex $X$-field hence plays two roles. First of all, the vev of its CP-even component breaks lepton number by two units generating neutrino masses via an inverse seesaw mechanism. Secondly, its CP-odd scalar component plays a crucial role to get the right thermal relic density.

In so far our paper differs from previous SUSY inverse seesaw models which did not discuss explicitly the origin of lepton-number violation. Most similar to our work we found are [19-21, 36, 38], where a heavy $B-L$ breaking Higgs or $Z^{\prime}$ resonance plays an important role for the DM annihilation cross section. However, the final state contains usually more SM particles compared to our model, which makes the phenomenology very different from ours.

\section{Masses of sneutrinos, neutralinos, and Higgs fields}

Before we discuss in detail DM phenomenology we first briefly discuss the spectrum of the relevant fields. The approximate formulas, which we present in this section, help a great deal in identifying the relevant parameter space.

For the convenience of the reader we recapitulate the superpotential of our model, cf. [4],

$$
\mathcal{W}=\mathcal{W}_{\mathrm{MSSM}}+\mathcal{W}_{\nu}
$$

where

$$
\begin{aligned}
\mathcal{W}_{\mathrm{MSSM}} & =Y_{u} \hat{Q} \hat{H}_{u} \hat{U}^{c}-Y_{d} \hat{Q} \hat{H}_{d} \hat{D}^{c}-Y_{e} \hat{L} \hat{H}_{d} \hat{E}^{c}+\mu_{H} \hat{H}_{u} \hat{H}_{d} \\
\mathcal{W}_{\nu} & =Y_{\nu} \hat{L} \hat{H}_{u} \hat{N}^{c}+\mu_{N S} \hat{N}^{c} \hat{S}+\frac{\lambda}{2} \hat{X} \hat{S}^{2}+\frac{\kappa}{3} \hat{X}^{3} .
\end{aligned}
$$


Beyond the MSSM Yukawa couplings and $\mu_{H}$-term we have three new Yukawa couplings $Y_{\nu}, \lambda$ and $\kappa$ and a new mass parameter $\mu_{N S}$.

Similarly the soft SUSY breaking terms can be grouped into an ordinary MSSM part and additional terms

$$
-\mathcal{L}_{\text {soft }}=-\mathcal{L}_{\text {soft,MSSM }}-\mathcal{L}_{\text {soft }, \nu}
$$

where

$$
\begin{aligned}
-\mathcal{L}_{\text {soft }, \mathrm{MSSM}}= & \frac{1}{2} M_{1} \tilde{B} \tilde{B}+\frac{1}{2} M_{2} \tilde{W} \tilde{W}+\frac{1}{2} M_{3} \tilde{g} \tilde{g} \\
& +M_{\tilde{Q}}^{2} \tilde{Q}^{\dagger} \tilde{Q}+M_{\tilde{U^{c}}}^{2} \tilde{U^{c}} \tilde{U}_{c}+M_{\tilde{D}^{c}}^{2} \tilde{D}^{c^{\dagger}} \tilde{D}_{c}+M_{\tilde{L}}^{2} \tilde{L}^{\dagger} \tilde{L}+M_{\tilde{E}^{c}}^{2} \tilde{E}^{c^{\dagger}} \tilde{E}^{c} \\
& +M_{H_{u}}^{2} H_{u}^{\dagger} H_{u}+M_{H_{d}}^{2} H_{d}^{\dagger} H_{d}+\left(b_{H} H_{u} H_{d}+\text { H.c. }\right) \\
& +\left(A_{u} \tilde{Q} H_{u} \tilde{U}^{c}-A_{d} \tilde{Q} H_{d} \tilde{D}^{c}-A_{e} \tilde{L} H_{d} \tilde{E}^{c}+\text { H.c. }\right) \\
-\mathcal{L}_{\text {soft }, \nu}= & M_{\tilde{N}^{c}}^{2} \tilde{N}^{c \dagger} \tilde{N}^{c}+M_{\tilde{S}}^{2} \tilde{S}^{\dagger} \tilde{S}+M_{X}^{2} X^{\dagger} X+\left(b_{N S} \tilde{N}^{c} \tilde{S}+\text { H.c. }\right) \\
& +\left(A_{\nu} \tilde{L} H_{u} \tilde{N}^{c}+\frac{1}{2} A_{\lambda} X \tilde{S}^{2}+\frac{1}{3} A_{\kappa} X^{3}+\text { H.c. }\right)
\end{aligned}
$$

For each new Yukawa coupling we have introduced a new trilinear coupling, $A_{\nu}, A_{\lambda}$ and $A_{\kappa}$. Furthermore, we have introduced $b_{N S}$ which corresponds to $\mu_{N S}$ in the superpotential and mass parameters for the new scalar fields, $M_{\tilde{N}^{c}}^{2}, M_{\tilde{S}}^{2}$ and $M_{X}^{2}$. For better readability we have suppressed here any flavor and gauge indices which can be easily reconciled from table 1.

\subsection{Sneutrinos}

We begin our discussion with the scalar partners of the neutrinos. Compared to the MSSM our model contains many more sneutrinos. As we had discussed in our previous paper [4] the leading-order expression for the sneutrino masses reads

$$
m_{\tilde{\nu}^{R}}^{2} \approx m_{\tilde{\nu}^{I}}^{2} \approx\left(\begin{array}{ccc}
\Re\left(M_{\tilde{L}}^{2}\right)+\frac{1}{2} M_{Z}^{2} \cos (2 \beta) & 0 & 0 \\
0 & \Re\left(M_{\tilde{N}^{c}}^{2}+\mu_{N S} \mu_{N S}^{\dagger}\right) & \Re\left(b_{N S}\right) \\
0 & \Re\left(b_{N S}^{T}\right) & \Re\left(M_{\tilde{S}}^{2}+\mu_{N S}^{\dagger} \mu_{N S}\right)
\end{array}\right) .
$$

In our numerical results later on, we use GUT-scale boundary conditions where all bilinear sfermion mass parameters are set to $m_{0}$ at the GUT scale, cf. next section. For simplicity and clarity we focus for the moment on the case of one generation of left-handed, righthanded and singlet scalars each. At the GUT scale the approximate mass matrix then further simplifies to

$$
m_{\tilde{\nu}^{R}}^{2} \approx m_{\tilde{\nu}^{I}}^{2} \approx\left(\begin{array}{ccc}
m_{0}^{2}+\frac{1}{2} M_{Z}^{2} \cos (2 \beta) & 0 & 0 \\
0 & m_{0}^{2}+\mu_{N S}^{2} & m_{0}^{2} \\
0 & m_{0}^{2} & m_{0}^{2}+\mu_{N S}^{2}
\end{array}\right)
$$

For $\mu_{N S}=0$ one sneutrino is strictly massless which might lead to phenomenological issues. Nevertheless, we assume $\mu_{N S}$ to be of the order of the electroweak scale, so we will not discuss this case any further. 
To get an estimate of the sneutrino masses at the electroweak scale we discuss now the renormalization group ( $R G$ ) corrections to the leading-order expression in eq. (3.7). For the one-loop $\beta$-functions we use the results calculated by SARAH $[41,42]$

$$
\begin{aligned}
\beta_{\mu_{N S}}^{(1)}= & 2 Y_{\nu} Y_{\nu}^{\dagger} \mu_{N S}+\mu_{N S} \lambda^{*} \lambda \\
\beta_{b_{N S}}^{(1)}= & 2 \mu_{N S} \lambda^{*} A_{\lambda}+2 Y_{\nu} Y_{\nu}^{\dagger} b_{N S}+4 A_{\nu} Y_{\nu}^{\dagger} \mu_{N S}+b_{N S} \lambda^{*} \lambda \\
\beta_{M_{\tilde{L}}^{2}}^{(1)}= & -\frac{6}{5} g_{1}^{2} \mathbf{1}\left|M_{1}\right|^{2}-6 g_{2}^{2} \mathbf{1}\left|M_{2}\right|^{2}+2 m_{H_{d}}^{2} Y_{e}^{\dagger} Y_{e}+2 m_{H_{u}}^{2} Y_{\nu}^{\dagger} Y_{\nu}+2 A_{e}^{\dagger} A_{e}+2 A_{\nu}^{\dagger} A_{\nu} \\
& +M_{\tilde{L}}^{2} Y_{e}^{\dagger} Y_{e}+M_{\tilde{L}}^{2} Y_{\nu}^{\dagger} Y_{\nu}+2 Y_{e}^{\dagger} M_{\tilde{E}}^{2} Y_{e}+Y_{e}^{\dagger} Y_{e} M_{\tilde{L}}^{2}+2 Y_{\nu}^{\dagger} M_{\tilde{N}}^{2} Y_{\nu}+Y_{\nu}^{\dagger} Y_{\nu} M_{\tilde{L}}^{2} \\
& -\frac{3}{5} g_{1}^{2} \mathbf{1}\left(-2 \operatorname{Tr}\left(M_{\tilde{U}}^{2}\right)-\operatorname{Tr}\left(M_{\tilde{L}}^{2}\right)-m_{H_{d}}^{2}+m_{H_{u}}^{2}+\operatorname{Tr}\left(M_{\tilde{D}}^{2}\right)+\operatorname{Tr}\left(M_{\tilde{E}}^{2}\right)+\operatorname{Tr}\left(M_{\tilde{Q}}^{2}\right)\right),
\end{aligned}
$$

$$
\begin{aligned}
\beta_{M_{\tilde{N}^{c}}^{2}}^{(1)} & =2\left(2 A_{\nu} A_{\nu}^{\dagger}+2 m_{H_{u}}^{2} Y_{\nu} Y_{\nu}^{\dagger}+2 Y_{\nu} M_{\tilde{L}}^{2} Y_{\nu}^{\dagger}+M_{\tilde{N}^{c}}^{2} Y_{\nu} Y_{\nu}^{\dagger}+Y_{\nu} Y_{\nu}^{\dagger} M_{\tilde{N}^{c}}^{2}\right), \\
\beta_{M_{\tilde{S}}^{2}}^{(1)} & =2 A_{\lambda}^{*} A_{\lambda}+2 \lambda^{*} M_{\tilde{S}}^{2 *} \lambda+2 M_{X}^{2} \lambda^{*} \lambda+M_{\tilde{S}^{2}}^{2} \lambda^{*} \lambda+\lambda^{*} \lambda M_{\tilde{S}}^{2} .
\end{aligned}
$$

We assume that all trilinear couplings are equal to the product of $A_{0}$ and the corresponding Yukawa coupling. To get the expression in eq. (3.7) we had neglected all terms proportional to some powers of the small expansion parameters $\epsilon$, cf. [4]. In particular, if we neglected terms proportional to some powers of $Y_{\nu}$ and $\lambda$, we could simplify the $\beta$-functions as well to

$$
\begin{aligned}
\beta_{\mu_{N S}=}^{(1)}= & \mathcal{O}\left(\epsilon^{2}\right), \\
\beta_{b_{N S}}^{(1)}= & \mathcal{O}\left(\epsilon^{2}\right), \\
\beta_{M_{\tilde{L}}^{2}}^{(1)}= & -\frac{6}{5} g_{1}^{2} \mathbf{1}\left|M_{1}\right|^{2}-6 g_{2}^{2} \mathbf{1}\left|M_{2}\right|^{2}+2 m_{H_{d}}^{2} Y_{e}^{\dagger} Y_{e}+2 A_{e}^{\dagger} A_{e}+M_{\tilde{L}}^{2} Y_{e}^{\dagger} Y_{e}+2 Y_{e}^{\dagger} M_{\tilde{E}}^{2} Y_{e}+Y_{e}^{\dagger} Y_{e} M_{\tilde{L}}^{2} \\
& -\frac{3}{5} g_{1}^{2} \mathbf{1}\left(-2 \operatorname{Tr}\left(M_{\tilde{U}}^{2}\right)-\operatorname{Tr}\left(M_{\tilde{L}}^{2}\right)-m_{H_{d}}^{2}+m_{H_{u}}^{2}+\operatorname{Tr}\left(M_{\tilde{D}}^{2}\right)+\operatorname{Tr}\left(M_{\tilde{E}}^{2}\right)+\operatorname{Tr}\left(M_{\tilde{Q}}^{2}\right)\right) \\
& +\mathcal{O}\left(\epsilon^{2}\right), \\
\beta_{M_{\tilde{N}^{c}}^{2}}^{(1)}= & \mathcal{O}\left(\epsilon^{2}\right), \\
\beta_{M_{\tilde{S}}^{(1)}}^{2}= & \mathcal{O}\left(\epsilon^{2}\right) .
\end{aligned}
$$

This implies that the mass matrix for the right-handed sneutrinos and scalar singlets is very well approximated by the high-scale boundary conditions. Only the left-handed sneutrino mass parameters have sizeable one-loop running, which is positive (at the low scale $\left.M_{\tilde{L}}^{2}>m_{0}^{2}\right)$.

The simplified expression for the sneutrino mass matrix in eq. (3.8) can be easily diagonalised with three eigenvalues $\mu_{N S}^{2}, \mu_{N S}^{2}+m_{0}^{2}$, and $M_{\tilde{L}}^{2}+\frac{1}{2} M_{Z}^{2} \cos (2 \beta)$. It is straightforward to write down the approximate eigenstates

$$
\tilde{\nu}_{1,2} \approx \frac{1}{\sqrt{2}}\left(\tilde{N}_{1}^{c} \mp \tilde{S}_{1}\right) \text { and } \tilde{\nu}_{3} \approx \tilde{L}_{1} .
$$

Since we know that the predominantly left-handed sneutrino DM has already been excluded by direct detection experiments [7, 10], sneutrinos can only be DM if it is mostly righthanded and/or scalar singlet, i.e., if $\mu_{N S}^{2} \lesssim m_{0}^{2}+M_{Z}^{2}$. In fact, we expect that the second 
lightest sneutrino in each generation is the left-handed one. The sneutrino DM candidate is then, to a good approximation, a maximally mixed superposition of the right-handed sneutrino and the scalar singlet. Strictly speaking we have two DM candidates since the real and the imaginary parts of the sneutrinos are very close in mass and both can contribute to the DM density. In fact, as we will see later on they both contribute to the relic density and we have a two component DM.

To understand under what conditions the real or imaginary component is the lighter one and when both states can co-annihilate, we discuss now the leading tree-level corrections to the masses of the lightest sneutrinos. Using ordinary perturbation theory it is straight-forward to find that

$$
m_{\tilde{\nu}_{1}}^{2} \approx \mu_{N S}^{2}+\langle 1|\delta M| 1\rangle
$$

where $\sqrt{2}|1\rangle=(0,1,-1)$ and $\delta M$ is

$$
\delta M_{R}=\left(\begin{array}{ccc}
\frac{1}{2} v_{u}^{2} Y_{\nu}^{2} & -\frac{Y_{\nu}}{\sqrt{2}}\left(v_{d} \mu_{H}-v_{u} A_{0}\right) & \frac{Y_{\nu}}{\sqrt{2}} v_{u} \mu_{N S} \\
-\frac{Y_{\nu}}{\sqrt{2}}\left(v_{d} \mu_{H}-v_{u} A_{0}\right) & \frac{1}{2} v_{u}^{2} Y_{\nu}^{2} & \frac{\lambda}{\sqrt{2}} v_{X} \mu_{N S} \\
\frac{Y_{\nu}}{\sqrt{2}} v_{u} \mu_{N S} & \frac{\lambda}{\sqrt{2}} v_{X} \mu_{N S} & \left.\frac{1}{2} \lambda v_{X}\left(\sqrt{2} A_{0}+(\kappa+\lambda) v_{X}\right)\right)
\end{array}\right)
$$

for the CP-even sneutrinos and

$$
\delta M_{I}=\left(\begin{array}{ccc}
\frac{1}{2} v_{u}^{2} Y_{\nu}^{2} & -\frac{Y_{\nu}}{\sqrt{2}}\left(v_{d} \mu_{H}-v_{u} A_{0}\right) & \frac{Y_{\nu}}{\sqrt{2}} v_{u} \mu_{N S} \\
-\frac{Y_{\nu}}{\sqrt{2}}\left(v_{d} \mu_{H}-v_{u} A_{0}\right) & \frac{1}{2} v_{u}^{2} Y_{\nu}^{2} & -\frac{\lambda}{\sqrt{2}} v_{X} \mu_{N S} \\
\frac{Y_{\nu}}{\sqrt{2}} v_{u} \mu_{N S} & -\frac{\lambda}{\sqrt{2}} v_{X} \mu_{N S} & \left.-\frac{1}{2} \lambda v_{X}\left(\sqrt{2} A_{0}+(\kappa-\lambda) v_{X}\right)\right)
\end{array}\right)
$$

for the CP-odd sneutrinos. Note that we do not distinguish here between the different ISS cases [4], which would further simplify the expressions if $Y_{\nu} \ll \lambda$ or $\lambda \ll Y_{\nu}$.

The corrections of the lightest sneutrino masses read

$$
\begin{aligned}
\left\langle 1\left|\delta M_{R}\right| 1\right\rangle & =\frac{Y_{\nu}^{2}}{4} v_{u}^{2}+\frac{1}{4} \lambda v_{X}\left(\sqrt{2} A_{0}-2 \sqrt{2} \mu_{N S}+(\kappa+\lambda) v_{X}\right), \\
\left\langle 1\left|\delta M_{I}\right| 1\right\rangle & =\frac{Y_{\nu}^{2}}{4} v_{u}^{2}-\frac{1}{4} \lambda v_{X}\left(\sqrt{2} A_{0}-2 \sqrt{2} \mu_{N S}+(\kappa-\lambda) v_{X}\right), \\
& \Rightarrow m_{\tilde{\nu}_{1}^{R}}^{2}-m_{\tilde{\nu}_{1}^{I}}^{2} \approx \frac{1}{2} \lambda v_{X}\left(\sqrt{2} A_{0}-2 \sqrt{2} \mu_{N S}+\kappa v_{X}\right) .
\end{aligned}
$$

The mass splitting between the two lightest sneutrinos is of the order of $\lambda M_{\mathrm{SUSY}}^{2}$ and vanishes for $v_{X}=0$ as expected.

\section{$3.2 \quad$ Neutralinos}

Technically speaking the Xino, $\tilde{X}$, is a neutralino since it has the same quantum numbers as the other MSSM neutralinos and will in general mix with them. On tree level the 

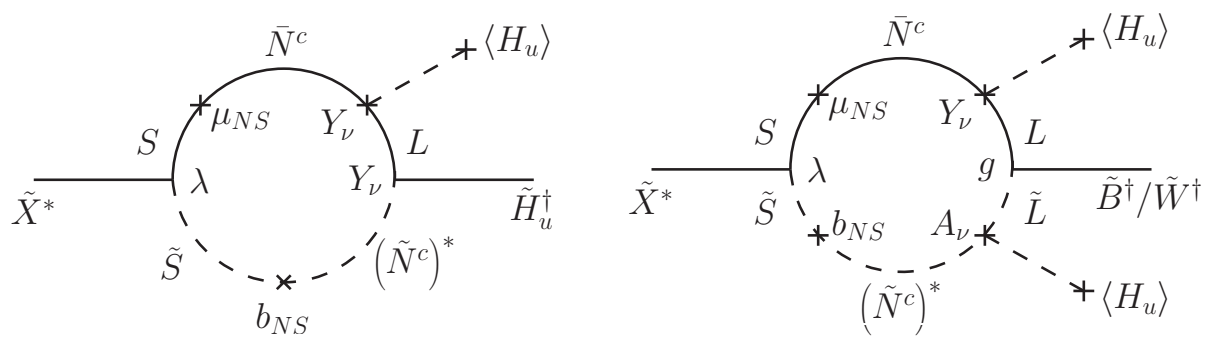

Figure 1. Typical one-loop diagrams inducing a mixing between the Xino and the MSSM neutralinos.

neutralino mass matrix in the basis $\left(\tilde{B}, \tilde{W}^{0}, \tilde{H}_{d}^{0}, \tilde{H}_{u}^{0}, \tilde{X}\right)$ has the structure

$$
M_{\tilde{\chi}^{0}}=\left(\begin{array}{ccccc}
M_{1} & 0 & -\frac{1}{2} g_{1} v_{d} & \frac{1}{2} g_{1} v_{u} & 0 \\
0 & M_{2} & \frac{1}{2} g_{2} v_{d} & -\frac{1}{2} g_{2} v_{u} & 0 \\
-\frac{1}{2} g_{1} v_{d} & \frac{1}{2} g_{2} v_{d} & 0 & -\mu_{H} & 0 \\
\frac{1}{2} g_{1} v_{u} & -\frac{1}{2} g_{2} v_{u} & -\mu_{H} & 0 & 0 \\
0 & 0 & 0 & 0 & \sqrt{2} \kappa v_{X}
\end{array}\right),
$$

which we have confirmed independently using SARAH $[41,42]$.

Since the mixing of the Xino with the other neutralinos is absent on tree-level we expect it to be small. In figure 1 we show typical one-loop diagrams which generate such mixings. Since all mass parameters are assumed to be of the order of the electroweak scale it is easy to estimate the size of the mixing terms

$$
\left(\delta M_{\tilde{\chi}^{0}}\right)_{i 5} \sim \frac{1}{16 \pi^{2}} \lambda Y_{\nu}^{2} \times \mathcal{O}(\mathrm{TeV})
$$

where we have assumed that $A_{\nu} \sim Y_{\nu} \times \mathcal{O}(\mathrm{TeV})$. Remembering that $m_{\nu} \sim Y_{\nu} \lambda Y_{\nu}^{T} \times \mathcal{O}(\mathrm{TeV})$ in our model [4] we see that the mixing terms are of the size of the neutrino masses suppressed by one additional loop factor which can be safely neglected. This is confirmed as well by our numerical results.

Let us comment briefly on the Xino properties. The Xino could also be the LSP depending on the parameter choice. However, its dominant annihilation channel into $A_{X} H_{X}$ has in general a too large cross section to give the right relic density because of the $\mathcal{O}(1)$ coupling $\kappa$. Only when the phase space for this process closes the annihilation cross section could be sufficiently suppressed but we do not discuss this possibility any further.

The other four neutralinos are just the ordinary, well-known MSSM neutralinos and we do not discuss them any further here.

\subsection{Higgs bosons}

The third sector, the Higgs sector, differs from that of the ordinary MSSM, by having two additional scalars. We decompose the scalar component of the superfield $\hat{X}$ as

$$
X=\frac{1}{\sqrt{2}}\left(v_{X}+\phi_{X}+\mathrm{i} \sigma_{X}\right) .
$$


Note that we have used here a different normalisation convention for the vev compared to our original paper [4] in order to directly use the results from SARAH [41, 42] without tedious checks for factors of $\sqrt{2}$. For all the results in this paper we have switched to the SARAH conventions.

The scalar mass matrix then reads on tree-level in the basis $\left(\phi_{d}, \phi_{u}, \phi_{X}\right)$ :

$$
m_{h}^{2}=\left(\begin{array}{ccc}
m_{\phi_{d} \phi_{d}}^{2} & -\frac{1}{4}\left(g_{1}^{2}+g_{2}^{2}\right) v_{d} v_{u}-\Re\left(b_{H}\right) & 0 \\
-\frac{1}{4}\left(g_{1}^{2}+g_{2}^{2}\right) v_{d} v_{u}-\Re\left(b_{H}\right) & m_{\phi_{u} \phi_{u}}^{2} & 0 \\
0 & 0 & m_{\phi_{X} \phi_{X}}^{2}
\end{array}\right),
$$

where

$$
\begin{aligned}
m_{\phi_{d} \phi_{d}}^{2} & =\frac{1}{8}\left(g_{1}^{2}+g_{2}^{2}\right)\left(3 v_{d}^{2}-v_{u}^{2}\right)+M_{H_{d}}^{2}+\left|\mu_{H}\right|^{2}, \\
m_{\phi_{u} \phi_{u}}^{2} & =-\frac{1}{8}\left(g_{1}^{2}+g_{2}^{2}\right)\left(-3 v_{u}^{2}+v_{d}^{2}\right)+M_{H_{u}}^{2}+\left|\mu_{H}\right|^{2}, \\
m_{\phi_{X} \phi_{X}}^{2} & =v_{X}\left(3 v_{X}|\kappa|^{2}+\sqrt{2} \Re\left(A_{\kappa}\right)\right)+M_{X}^{2} .
\end{aligned}
$$

The fields $\phi_{d}$ and $\phi_{u}$ are the CP-even and electrically neutral components of the MSSM Higgs doublets $H_{d}$ and $H_{u}$, respectively. Similarly the fields $\sigma_{d}$ and $\sigma_{u}$ are their CP-odd components and the mass matrix for the CP-odd Higgs-like scalars reads on tree-level in the basis $\left(\sigma_{d}, \sigma_{u}, \sigma_{X}\right)$ :

$$
m_{A^{0}}^{2}=\left(\begin{array}{ccc}
m_{\sigma_{d} \sigma_{d}}^{2} & \Re\left(b_{H}\right) & 0 \\
\Re\left(b_{H}\right) & m_{\sigma_{u} \sigma_{u}}^{2} & 0 \\
0 & 0 & m_{\sigma_{X} \sigma_{X}}^{2}
\end{array}\right)
$$

where

$$
\begin{aligned}
m_{\sigma_{d} \sigma_{d}}^{2} & =\frac{1}{8}\left(g_{1}^{2}+g_{2}^{2}\right)\left(-v_{u}^{2}+v_{d}^{2}\right)+M_{H_{d}}^{2}+\left|\mu_{H}\right|^{2}, \\
m_{\sigma_{u} \sigma_{u}}^{2} & =-\frac{1}{8}\left(g_{1}^{2}+g_{2}^{2}\right)\left(-v_{u}^{2}+v_{d}^{2}\right)+M_{H_{u}}^{2}+\left|\mu_{H}\right|^{2}, \\
m_{\sigma_{X} \sigma_{X}}^{2} & =v_{X}\left(-\sqrt{2} \Re\left(A_{\kappa}\right)+v_{X}|\kappa|^{2}\right)+M_{X}^{2},
\end{aligned}
$$

and we have neglected gauge fixing contributions.

Similar to the Xino and the MSSM neutralinos, our new scalars do not mix with the MSSM Higgs fields at tree-level and the loop-level mixing is negligibly small using similar arguments as for the Xino case. Hence, we label the new scalars as follows $\phi_{X} \equiv H_{X}$ and $\sigma_{X} \equiv A_{X}$, which are mass and symmetry eigenstates simultaneously to a very good approximation. Note that $M_{X}^{2}$ is fixed by the tadpole condition

$$
\begin{aligned}
\frac{\partial V}{\partial \phi_{X}} & =\frac{v_{X}^{2}}{\sqrt{2}} \Re\left(A_{\kappa}\right)+M_{X}^{2} v_{X}+v_{X}^{3}|\kappa|^{2}=0 \\
& \Leftrightarrow M_{X}^{2}=-\frac{v_{X}}{\sqrt{2}} \Re\left(A_{\kappa}\right)-v_{X}^{2}|\kappa|^{2} .
\end{aligned}
$$


We can use this in the formulas for the scalar masses

$$
\begin{aligned}
& m_{H_{X}}^{2}=2|\kappa|^{2} v_{X}^{2}+\frac{v_{X}}{\sqrt{2}} \Re\left(A_{\kappa}\right), \\
& m_{A_{X}}^{2}=-\frac{3 v_{X}}{\sqrt{2}} \Re\left(A_{\kappa}\right) .
\end{aligned}
$$

Before we quantify this equations further, we have to comment on the running of $A_{\kappa}$ which is significant. The relevant one-loop $\beta$-functions are given by

$$
\begin{aligned}
\beta_{\kappa} & =6 \kappa|\kappa|^{2}+\frac{3}{2} \kappa \operatorname{Tr}\left(\lambda \lambda^{*}\right) \approx 6 \kappa|\kappa|^{2} \\
\beta_{A_{\kappa}} & =18|\kappa|^{2} A_{\kappa}+3 \kappa \operatorname{Tr}\left(\lambda^{*} A_{\lambda}\right)+\frac{3}{2} A_{\kappa} \operatorname{Tr}\left(\lambda \lambda^{*}\right) \approx 18|\kappa|^{2} A_{\kappa} .
\end{aligned}
$$

Neglecting the small terms proportional to some powers of $\lambda$ we can solve this set of coupled differential equations analytically and find

$$
\begin{aligned}
\kappa(\mu) & =\frac{\kappa_{0}}{\sqrt{1+\frac{3 \kappa_{0}^{2}}{4 \pi^{2}} \log \left(M_{\mathrm{SUSY}} / \mu\right)}} \\
A_{\kappa}(\mu) & =\kappa_{0} A_{0}\left(\frac{\left(1+\frac{3 \kappa_{0}^{2}}{4 \pi^{2}} \log \left(M_{\mathrm{SUSY}} / M_{\mathrm{GUT}}\right)\right)^{2}}{\left(1+\frac{3 \kappa_{0}^{2}}{4 \pi^{2}} \log \left(M_{\mathrm{SUSY}} / \mu\right)\right)^{3}}\right)^{\frac{1}{2}}
\end{aligned}
$$

where $\kappa_{0}=\kappa\left(M_{\mathrm{SUSY}}\right)$. At the low scale where we want to evaluate the scalar masses

$$
A_{\kappa}\left(M_{\mathrm{SUSY}}\right)=\kappa_{0} A_{0}\left(1+\frac{3 \kappa_{0}^{2}}{4 \pi^{2}} \log \left(M_{\mathrm{SUSY}} / M_{\mathrm{GUT}}\right)\right) \approx \kappa_{0} A_{0}\left(1-2.3 \kappa_{0}^{2}\right)
$$

where we have used $M_{\mathrm{GUT}} \approx 2 \times 10^{16} \mathrm{GeV}$ and $M_{\mathrm{SUSY}} \approx 10^{3} \mathrm{GeV}$. At the SUSY scale we find for the RG corrected scalar masses

$$
\begin{aligned}
& m_{H_{X}}^{2} \approx 2 \kappa_{0}^{2} v_{X}^{2}+\frac{v_{X}}{\sqrt{2}} \kappa_{0} A_{0}\left(1-2.3 \kappa_{0}^{2}\right), \\
& m_{A_{X}}^{2} \approx-\frac{3 v_{X}}{\sqrt{2}} \kappa_{0} A_{0}\left(1-2.3 \kappa_{0}^{2}\right) .
\end{aligned}
$$

To avoid the new scalars becoming tachyonic we find a simple constraint on $A_{0}$

$$
-\frac{2 \sqrt{2} \kappa_{0}}{1-2.3 \kappa_{0}^{2}} v_{X} \lesssim A_{0}<0
$$

We checked the approximate formulas for the scalar masses and found that they are correct up to a few percent, cf. figure 2. In particular, $H_{X}$ receives corrections from finite loop corrections, which we do not discuss here in detail. Note that the mass ordering of $A_{X}$ and $H_{X}$ is not fixed, but depends on $A_{0}$. This insight helps to separate the $A_{X}$-funnel from the $H_{X}$-funnel region. 


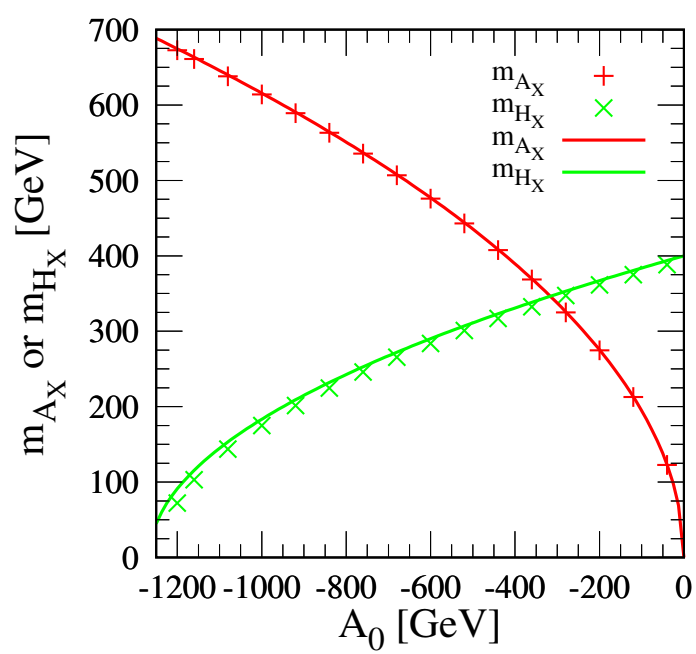

Figure 2. Comparison of the analytic results (straight lines) for the masses of $H_{X}$ and $A_{X}$ (eqs. (3.46) and (3.47)) with numerical SPheno results (crosses) as a function of $A_{0}$. Here, we take $\kappa=0.4$ and $\sqrt{2} v_{X}=10^{3} \mathrm{GeV}$.

\section{Sneutrino dark matter}

In this section we discuss the sneutrino as a DM candidate and focus on (co-)annihilation channels which are unique to our model. One of them can overcome issues of having a right-handed sneutrino as a thermally produced DM candidate [14]. In some sense our approach is similar to the one advertised in [17], but we do not introduce a new gauge interaction. We only introduce a new complex scalar which breaks a discrete symmetry and no new vector fields.

This new $X$-scalar splits into a CP-even and a CP-odd scalar after symmetry breaking such that the new $X$-funnel consists in fact out of two channels, the $H_{X}$-funnel and the $A_{X}$-funnel. The real and imaginary parts of the lightest sneutrino can then (co-)annihilate via these channels. However, as we will show the cross section is large enough only for the $A_{X}$-funnel to realize the usual freeze-out mechanism. The pseudoscalar $A_{X}$ couples predominantly to the heavy neutrinos, which then decay further into SM particles before big bang nucleosynthesis.

We focus here mainly on these channels since they are unique to our model. Other channels like the MSSM Higgs funnels might work as well to realize sneutrino DM, but these channels have been well studied before, e.g., [43].

\subsection{Numerical calculation of the particle spectra}

Before we discuss our results for these channels we describe how we determine the particle spectra. We have assumed GUT-scale boundary conditions with, in MSSM notation,

$$
\begin{aligned}
m_{0}^{2} & =\frac{1}{9} m_{\tilde{Q}}^{2}=\frac{1}{9} m_{\tilde{D}}^{2}=\frac{1}{9} m_{\tilde{U}}^{2}=m_{\tilde{L}}^{2}=m_{\tilde{E}}^{2}=m_{\tilde{N}}^{2}=m_{\tilde{S}}^{2}=m_{H_{u}}^{2}=m_{H_{d}}^{2}=b_{N S}, \\
M_{1 / 2} & =\frac{1}{3} M_{3}=M_{2}=M_{1}, \\
A_{i} & =A_{0} Y_{i}, A_{\lambda}=A_{0} \lambda, A_{\kappa}=\kappa A_{0},
\end{aligned}
$$




\begin{tabular}{|ccc|}
\hline Parameter & $A_{X}$-funnel & $H_{X^{-}}$funnel \\
\hline$m_{0}$ & $1 \mathrm{TeV}$ & $1 \mathrm{TeV}$ \\
$M_{1 / 2}$ & $1.3 \mathrm{TeV}$ & $1.3 \mathrm{TeV}$ \\
$A_{0}$ & $-250 \leq A_{0} / \mathrm{GeV} \leq-50$ & $-1200<A_{0} / \mathrm{GeV}<-350$ \\
$\tan \beta$ & 10 & 10 \\
$v_{X}$ & $1000 / \sqrt{2} \mathrm{GeV}$ & $1000 / \sqrt{2} \mathrm{GeV}$ \\
$\kappa_{0}$ & 0.4 & 0.4 \\
\hline$\lambda_{11}=0.5 \lambda_{22}$ & $1 \times 10^{-4}<\lambda_{11}<0.01$ & $1 \times 10^{-4}<\lambda_{11}<0.01$ \\
$c$ & $\{0.97,0.99\}$ & $\{0.97,0.99\}$ \\
\hline
\end{tabular}

Table 2. Parameters used in our numerical scans. We take $\mu_{N S}$ and $\lambda$ to be diagonal matrices. For more details, in particular on how we determine $\mu_{N S}$ see main text.

inspired by the constrained MSSM. To avoid the LHC constraints, we have set an arbitrary factor of 3 for the colored states in order to make them heavy enough. The $M_{X}^{2}$ is fixed at low scale by the tadpole condition as described in section 3.3. We treat $\tan \beta, v_{X}, \kappa$, $\lambda$, and $\mu_{N S}$ as free low-scale input parameters and for the sake of simplicity we choose $\lambda$ and $\mu_{N S}$ to be diagonal. We also set $\left(\mu_{N S}\right)_{22}=2\left(\mu_{N S}\right)_{11}$ and $\lambda_{22}=2 \lambda_{11}$ such that we can consider only one generation of sneutrinos effectively in our scans. In our model $\kappa$ is an order one parameter and we fix it to the reference value 0.4 . The reference value for the vev $v_{X}$ is chosen such that the Xino mass is $400 \mathrm{GeV}$ and our additional fermions and scalars are at the electroweak scale.

The neutrino Yukawa matrix $Y_{\nu}$ is in principle free as well, but we fix it using the tree-level formula

$$
Y_{\nu}=\frac{\mathrm{i}}{v_{u}} U_{\mathrm{PMNS}} \sqrt{m_{i}} \Omega\left(\sqrt{M_{S}^{d}}\right)^{-1} V_{S} \mu_{N S}
$$

for more details, see ref. [4]. We have used the latest results for normal ordered neutrinos from NuFIT [44], $m_{1}=0 \mathrm{eV}$ and set all CP-violating phases to zero to get real numerical values for $Y_{\nu}$. Note that our results depend only very weakly on the details of $Y_{\nu}$, but it is important to fix the order of the neutrino Yukawa couplings.

The calculation of the SUSY spectrum including two-loop corrections is taken care of by SPheno $[45,46]$. The necessary code is generated by SARAH $[41,42]$. In table 2, we give our benchmark parameter ranges for both funnels which we use in our numerical scans.

It is numerically very challenging to find the resonance region unless one understands where to look for it. In our scans we hence implement an iterative procedure which we describe first for the $A_{X}$-funnel. Apart from the fixed parameters in table 2 we first fix $\lambda_{11}$ and $A_{0}$ to get an estimate for $m_{A_{X}}$ using eq. (3.47). Then we choose the parameter $c \in\{0.97,0.99\}$ which controls how close the data point is to the resonance

$$
m_{\tilde{\nu}_{1}^{R}}+m_{\tilde{\nu}_{1}^{I}}=c m_{A_{X}}
$$




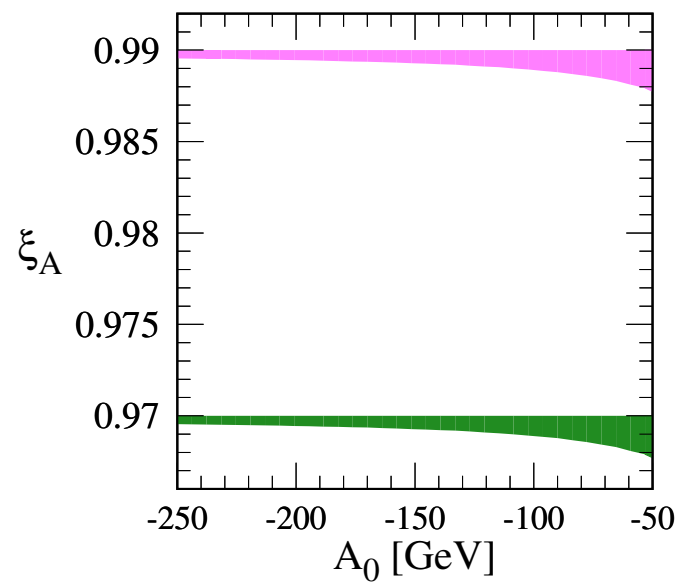

Figure 3. Graphical presentation of our resonance condition eq. (4.7). The upper line is for $c=0.99$ while the lower line is for $c=0.97$. The ratio $\xi_{A}$ deviates less than $2.5 \times 10^{-3}$ from the input value of $c$.

We then solve this equation with the formulas (3.20), (3.23), and (3.24) from section 3.1 to get an initial estimate for $\left(\mu_{N S}\right)_{11}$ :

$$
\begin{aligned}
\left(\mu_{N S}\right)_{11}= & \frac{c m_{A_{X}} \sqrt{\lambda_{11}^{2} v_{X}^{2}\left(2 A_{0}^{2}+2 \sqrt{2} A_{0} \kappa v_{X}+\left(\kappa^{2}+2 \lambda_{11}^{2}\right) v_{X}^{2}\right)+4 c^{4} m_{A_{X}}^{4}-6 c^{2} \lambda_{11}^{2} m_{A_{X}}^{2} v_{X}^{2}}}{4 c^{2} m_{A_{X}}^{2}-2 \lambda_{11}^{2} v_{X}^{2}} \\
& -\frac{2 A_{0} \lambda_{11}^{2} v_{X}^{2}+\sqrt{2} \kappa \lambda_{11}^{2} v_{X}^{3}}{8 c^{2} m_{A_{X}}^{2}-4 \lambda_{11}^{2} v_{X}^{2}} .
\end{aligned}
$$

Given all the initially estimated parameters, we then run SPheno to calculate a consistent spectrum. From this calculated spectrum, we take $m_{A_{X}}$ as reference mass to calculate again $\left(\mu_{N S}\right)_{11}$ from eq. (4.6), which we do three times. In our final run, we find that the ratio

$$
\xi_{A}=\frac{m_{\tilde{\nu}_{1}^{R}}+m_{\tilde{\nu}_{1}^{I}}}{m_{A_{X}}},
$$

deviates not more than $2.5 \times 10^{-3}$ from the input value $c$, see also figure 3 .

To keep the validity of our approximation we have set the constraint $A_{0}<-50 \mathrm{GeV}$. For larger and larger $A_{0}$, the pseudoscalar mass $m_{A_{X}}$ and hence our sneutrino masses have to become smaller and smaller. This implies that $\left(\mu_{N S}\right)_{11}$ should become naively smaller but then the correction term

$$
\frac{m_{\tilde{\nu}_{1}^{R}}^{2}-m_{\tilde{\nu}_{1}^{I}}^{2}}{m_{\tilde{\nu}_{1}^{R}}^{2}+m_{\tilde{\nu}_{1}^{I}}^{2}} \approx \frac{\lambda_{11} v_{X}\left(\sqrt{2} A_{0}-2 \sqrt{2}\left(\mu_{N S}\right)_{11}+\kappa v_{X}\right)}{4\left(\mu_{N S}\right)_{11}^{2}} \approx \frac{\lambda_{11} \kappa v_{X}^{2}}{4\left(\mu_{N S}\right)_{11}^{2}},
$$

is not small anymore since $v_{X}^{2} \gg\left(\mu_{N S}\right)_{11}^{2}$ which compensates the smallness of $\lambda_{11}$. This can also be seen in figure 3 where $\xi_{A}$ deviates more strongly from $c$ for larger $A_{0}$.

For the $H_{X}$-funnel we could follow basically the same approach but the relevant condition to estimate $\left(\mu_{N S}\right)_{11}$ is modified as

$$
2 m_{\chi}=c m_{H_{X}},
$$



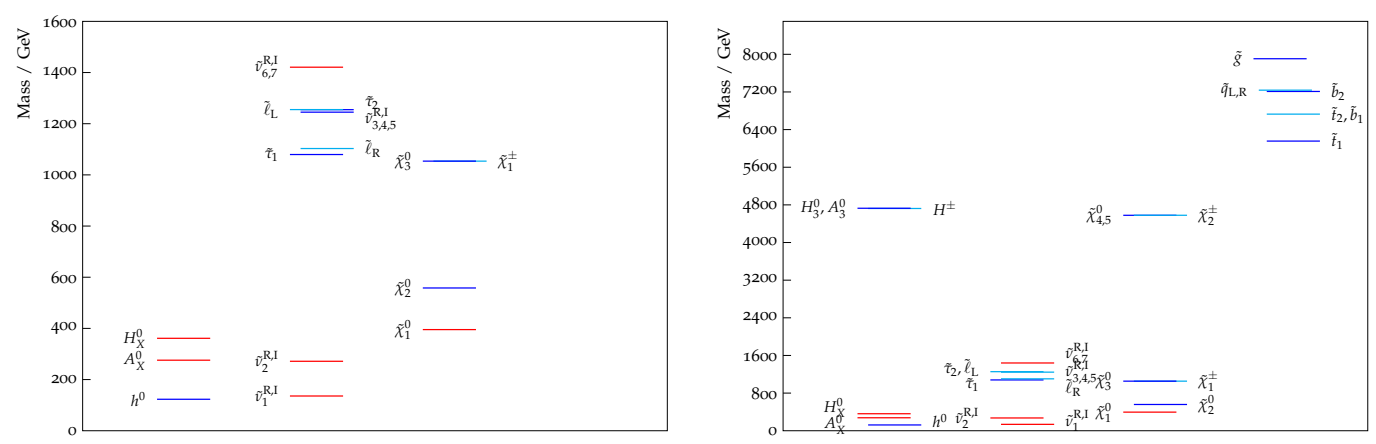

Figure 4. The SUSY mass spectrum of a typical $A_{X}$-resonance model point. The right panel shows the full spectrum and the left hand panel is zoomed into the mass scale between 0 to 1.6 $\mathrm{TeV}$. The red lines present the particles beyond the MSSM while blue lines represent the MSSM particles. Note that the cyan lines represent degenerate MSSM particles.

with an estimate for $\left(\mu_{N S}\right)_{11}$ :

$$
\left(\mu_{N S}\right)_{11}=\frac{\lambda_{11} v_{X}}{2 \sqrt{2}}+\frac{1}{2} \sqrt{c^{2} m_{H_{X}}^{2}-\frac{1}{2} \lambda_{11} v_{X}\left(2 \sqrt{2} A_{0}+2 \kappa v_{X}+\lambda_{11} v_{X}\right)} .
$$

The resonance parameter in this case reads

$$
\xi_{H}=\frac{2 m_{\chi}}{m_{H_{X}}},
$$

which we do not show since we will not use it later explicitly. In the $H_{X}$-funnel parameter set the lightest CP-even sneutrino is always our DM candidate since it is lighter than the CP-odd sneutrino, i.e. $m_{\chi}=m_{\tilde{\nu}_{1}^{R}}$.

With this procedure we have fixed now two sets of particle spectra which can be used in the further calculations.

Last but not least, we present a typical mass spectrum of an $A_{X}$-resonance model point in figure 4. We show its full spectrum in the right panel and a zoom-in for smaller masses between 0 to $1.6 \mathrm{TeV}$ in the left panel. We use red to represent the particles beyond the MSSM and blue for the MSSM particles. Sometimes the masses are very close to each other. In this case, we use cyan. Note that the MSSM colored particles such as squarks and gluino are heavy enough to evade the LHC constraints. Furthermore, the model is very safe from the monojet constraints as back-of-the-envelope calculations show that the rates are extremely tiny.

\section{2 (Co-)annihilation cross sections and relic density}

In our setup, the lightest sneutrino is mostly a maximally mixed combination of a righthanded sneutrino $\tilde{N}^{c}$ and the scalar component of the singlet superfield $\hat{S}$, cf. 3.1. The dominant (co-)annihilation channel in the early Universe for them is into neutrinos via the $A_{X^{-}}$or $H_{X}$-funnel, see figure 5 . Since we assume CP to be conserved $A_{X}$ couples to the real and imaginary part of the sneutrino while $H_{X}$ to the real part or the imaginary part of the sneutrino. This implies that the $A_{X}$-funnel is a co-annihilation channel while $H_{X}$ 

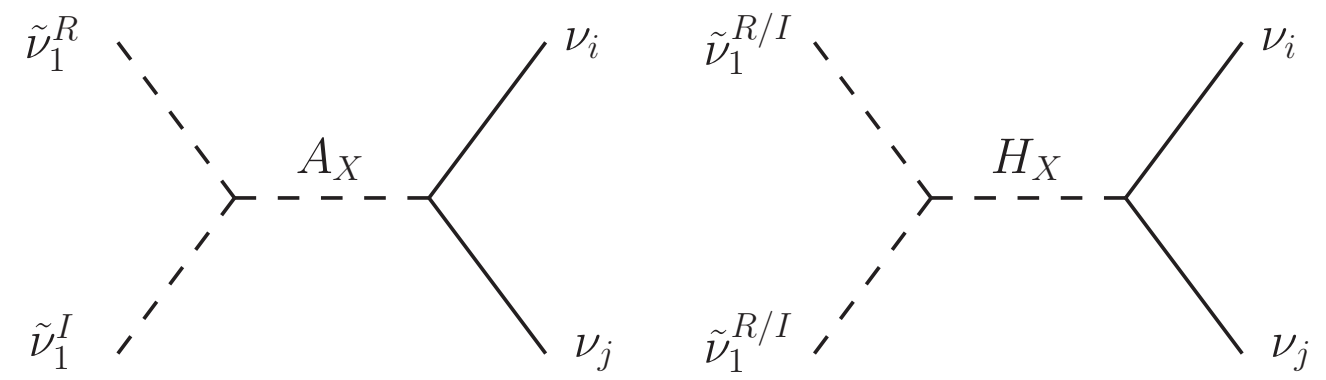

Figure 5. The relevant DM annihilation channel for $A_{X}$-funnel (left) and $H_{X}$-funnel (right) where $i, j=4,5$. Note that it turns out, that only the $A_{X}$-funnel is phenomenologically viable in our setup.

is an annihilation channel. In this subsection, we first present the co-annihilation via the $A_{X}$-funnel (scenario A) and then discuss why the annihilation via the $H_{X}$-funnel (scenario $\mathrm{H})$ is phenomenologically not viable.

\subsubsection{Scenario A: sneutrino co-annihilation via the $A_{X}$-funnel}

We begin our discussion with the $A_{X}$-funnel where $\tilde{\nu}_{1}^{R}$ and $\tilde{\nu}_{1}^{I}$ co-annihilate resonantly into neutrinos. It is worth to mention that in general resonances can be difficult to handle numerically. Furthermore, since our case also has co-annihilation, we will start to show complete and approximated formulae for complementarity. We have calculated the relic density by using MicrOMEGAs for cross checking and we found our relic density computation only differs with MicrOMEGAs 9\%-10\% which is properly taken into account by our systematic uncertainties. Hence, we perform relic density calculations by ourselves using SPheno $[45,46]$ for the particle spectrum, as already described in section 4.1.

The co-annihilation cross section into two heavy neutrino states reads

$$
\sigma(s)=\sum_{i, j=4}^{5} \frac{\left|C_{A \tilde{\nu} \tilde{\nu}}\right|^{2}\left|C_{A \nu \nu}\right|^{2}\left[s-\left(\left|m_{\nu_{i}}\right|-\left|m_{\nu_{j}}\right|\right)^{2}\right] \mathcal{S}}{8 \pi s\left[\left(s-m_{A_{X}}^{2}\right)^{2}+m_{A_{X}}^{2} \Gamma_{A_{X}}^{2}\right]} \sqrt{\frac{\left(s-m_{\nu_{j}}^{2}\right)^{2}-2 m_{\nu_{i}}^{2}\left(s+m_{\nu_{j}}^{2}\right)+m_{\nu_{i}}^{4}}{\left(s-m_{\tilde{\nu}_{1}^{R}}^{2}\right)^{2}-2 m_{\tilde{\nu}_{1}^{I}}^{2}\left(s+m_{\tilde{\nu}_{1}^{R}}^{2}\right)+m_{\tilde{\nu}_{1}^{I}}^{4}}},
$$

where $s=\left(p_{\tilde{\nu}_{1}^{R}}+p_{\tilde{\nu}_{1}^{I}}\right)^{2}=\left(p_{\nu_{i}}+p_{\nu_{j}}\right)^{2}$ and

$$
\mathcal{S}=\left\{\begin{array}{l}
1 \text { for } i \neq j \\
\frac{1}{2} \text { for } i=j
\end{array}\right.
$$

is a symmetry factor. We have taken the absolute values of $m_{\nu_{i}}$ and $m_{\nu_{j}}$ to emphasize that these are the positive physical masses. Here we have three choices for the decay channel, $(i, j)=(4,4),(4,5)$, and $(5,5)$. The direct annihilation into light active neutrinos is negligibly small. Note that the mass difference between the two heavy neutrinos is small 
compared to the mass itself. $C_{A \tilde{\nu} \tilde{\nu}}$ is the coupling of the sneutrinos to $A_{X}$

$$
\begin{aligned}
C_{A \tilde{\nu} \tilde{\nu}}= & -\mathrm{i}\left[\kappa v_{X} \lambda_{11} Z_{16}^{R} Z_{16}^{I} Z_{23}^{A}-\frac{\sqrt{2}}{2}\left(A_{\lambda}\right)_{11} Z_{16}^{R} Z_{16}^{I} Z_{23}^{A}\right. \\
& \left.-\frac{\sqrt{2}}{2} \lambda_{11}\left(\mu_{N S}\right)_{11} Z_{14}^{R} Z_{16}^{I} Z_{23}^{A}-\frac{\sqrt{2}}{2} \lambda_{11}\left(\mu_{N S}\right)_{11} Z_{16}^{R} Z_{14}^{I} Z_{23}^{A}\right] \\
\approx & -\mathrm{i} \frac{\lambda_{11}}{2} m_{A_{X}}\left(\frac{\kappa v_{X}}{m_{A_{X}}}-\frac{1}{\sqrt{2}} \frac{A_{0}}{m_{A_{X}}}+\sqrt{2} \frac{\left(\mu_{N S}\right)_{11}}{m_{A_{X}}}\right) \\
\approx & -\mathrm{i} \frac{\lambda_{11}}{c} m_{\chi}\left(\frac{c \kappa v_{X}}{2 m_{\chi}}+\frac{2}{3 c} \frac{m_{\chi}}{\kappa v_{X}}+\frac{c}{\sqrt{2}}\right),
\end{aligned}
$$

with the mixing matrices of real (imaginary) part of sneutrinos $Z^{R(I)}$ and pseudo-scalars $Z^{A}$ which are $Z_{14}^{R(I)}=-Z_{16}^{R(I)} \approx 1 / \sqrt{2}$ and $\left|Z_{23}^{A}\right|=1$, respectively. For our numerical results we use the full formulas, but to understand our results it is useful to look at approximate results as well.

Furthermore, $C_{A \nu \nu}$ corresponding to the coupling of $A_{X}$ to the neutrinos is given as

$$
C_{A \nu \nu}=-\frac{1}{\sqrt{2}}\left(\lambda_{11} U_{i 6}^{V} U_{j 6}^{V}+\lambda_{22} U_{i 7}^{V} U_{j 7}^{V}\right) Z_{23}^{A} \approx-\frac{\lambda_{11}}{2 \sqrt{2}},
$$

with the mixing matrices of neutrinos $U^{V}$ and we have used that $U_{i 6}^{V} \approx-1 / \sqrt{2} \gg U_{i 7}^{V}$ which shows that $C_{A \nu \nu}$ is of the order of $\lambda_{11}$. The total decay width of $A_{X}$ is dominated by the decay channels into heavy neutrinos, $\nu_{4,5}$ or DM which can be expressed as

$$
\left.\left.\Gamma_{A_{X}}\right|_{\text {tot }} \simeq \Gamma_{A_{X}}\right|_{A_{X} \rightarrow 2 \nu_{4}}+\left.\Gamma_{A_{X}}\right|_{A_{X} \rightarrow 2 \nu_{5}}+\left.\Gamma_{A_{X}}\right|_{A_{X} \rightarrow \nu_{4} \nu_{5}}+\left.\Gamma_{A_{X}}\right|_{A_{X} \rightarrow \tilde{\nu}_{1}^{R} \tilde{\nu}_{1}^{I}},
$$

where we have again neglected the decays into active neutrinos. Each term is calculated as follows

$$
\begin{aligned}
\left.\Gamma_{A_{X}}\right|_{A_{X} \rightarrow 2 \nu_{4}} & =\frac{1}{32 \pi} \lambda_{11}^{2}\left(U_{46}^{V}\right)^{4} m_{A_{X}} \sqrt{1-4\left(\frac{m_{\nu_{4}}}{m_{A_{X}}}\right)^{2}} \\
\left.\Gamma_{A_{X}}\right|_{A_{X} \rightarrow 2 \nu_{5}} & =\left.\Gamma_{A_{X}}\right|_{A_{X} \rightarrow 2 \nu_{4}}(4 \rightarrow 5) \\
\left.\Gamma_{A_{X}}\right|_{A_{X} \rightarrow \nu_{4} \nu_{5}} & =\frac{1}{16 \pi} \lambda_{11}^{2}\left(U_{46}^{V} U_{56}^{V}\right)^{2} m_{A_{X}}\left[1-\frac{\left(m_{\nu_{5}}-m_{\nu_{4}}\right)^{2}}{m_{A_{X}}^{2}}\right]^{3 / 2} \sqrt{1-\frac{\left(m_{\nu_{5}}+m_{\nu_{4}}\right)^{2}}{m_{A_{X}}^{2}}} \\
\left.\Gamma_{A_{X}}\right|_{A_{X} \rightarrow \tilde{\nu}_{1}^{R} \tilde{\nu}_{1}^{I}} & =\frac{1}{16 \pi} \frac{\left|C_{A \tilde{\nu} \tilde{\nu}}\right|^{2}}{m_{A_{X}}} \sqrt{1-\frac{\left(m_{\tilde{\nu}_{1}^{R}}-m_{\tilde{\nu}_{1}^{I}}\right)^{2}}{m_{A_{X}}^{2}}} \sqrt{1-\frac{\left(m_{\tilde{\nu}_{1}^{R}}+m_{\tilde{\nu}_{1}^{I}}\right)^{2}}{m_{A_{X}}^{2}}}
\end{aligned}
$$

Using the above equations the total decay width can be approximated by

$$
\left.\Gamma_{A_{X}}\right|_{\text {tot }} \approx \frac{\lambda_{11}^{2}}{64 \pi} m_{A_{X}} \sqrt{1-c^{2}} \times\left(2+\tilde{C}_{A \tilde{\nu} \tilde{\nu}}^{2}\right)=\frac{\lambda_{11}^{2}}{32 \pi} \frac{m_{\chi}}{c} \sqrt{1-c^{2}} \times\left(2+\tilde{C}_{A \tilde{\nu} \tilde{\nu}}^{2}\right),
$$


where $\tilde{C}_{A \tilde{\nu} \tilde{\nu}}^{2}$ is an $\mathcal{O}(1)$ factor given by

$$
\tilde{C}_{A \tilde{\nu} \tilde{\nu}}^{2} \equiv\left(\frac{\kappa v_{X}}{m_{A_{X}}}-\frac{1}{\sqrt{2}} \frac{A_{0}}{m_{A_{X}}}+\sqrt{2} \frac{\left(\mu_{N S}\right)_{11}}{m_{A_{X}}}\right)^{2} \approx\left(\frac{c \kappa v_{X}}{2 m_{\chi}}+\frac{2}{3 c} \frac{m_{\chi}}{\kappa v_{X}}+\frac{c}{\sqrt{2}}\right)^{2} .
$$

Note that this factor depends mildly on $m_{\chi}$. We can therefore simplify the thermal averaged cross section at the temperature $T[47]$ to

$$
\begin{aligned}
\langle\sigma v\rangle_{\mathrm{th}}^{A} & =\sum_{i, j=4}^{5} \frac{\left|C_{A \tilde{\nu} \tilde{\nu}}\right|^{2}\left|C_{A \nu \nu}\right|^{2} m_{A_{X}}^{2} \mathcal{S}}{64 m_{\chi}^{4} \Gamma_{A_{X}} T} \sqrt{\left(1-\frac{\left(m_{\tilde{\nu}_{1}^{R}}+m_{\tilde{\nu}_{1}^{I}}\right)^{2}}{m_{A_{X}}^{2}}\right)\left(1-\frac{\left(m_{\tilde{\nu}_{1}^{R}}-m_{\tilde{\nu}_{1}^{I}}\right)^{2}}{m_{A_{X}}^{2}}\right)^{-1}} \\
& \times \sqrt{\left(1-\frac{\left(m_{\nu_{i}}+m_{\nu_{j}}\right)^{2}}{m_{A_{X}}^{2}}\right)\left(1-\frac{\left(m_{\nu_{i}}-m_{\nu_{j}}\right)^{2}}{m_{A_{X}}^{2}}\right)^{3 / 2} \frac{K_{1}\left(\frac{m_{A_{X}}}{T}\right)}{K_{2}\left(\frac{m_{\tilde{\nu}_{1}^{R}}}{T}\right) K_{2}\left(\frac{m_{\tilde{\nu}_{1}^{I}}}{T}\right)},}
\end{aligned}
$$

with defining $m_{\chi} \equiv \min \left\{m_{\tilde{\nu}_{1}^{R}}, m_{\tilde{\nu}_{1}^{I}}\right\}$ as the DM mass. The functions $K_{n}(x), n=1,2$, are the modified Bessel functions of the second kind. Here, we have used the narrow width approximation which makes the $s$-integral easier since the propagator part can be replaced by $\pi /\left(m_{A_{X}} \Gamma_{A_{X}}\right) \delta\left(s-m_{A_{X}}^{2}\right)$. The total decay width $\Gamma_{A_{X}}$ is estimated in eq. (4.16). This is a complicated formula, but using our previous approximations we find

$$
\begin{aligned}
\langle\sigma v\rangle_{\mathrm{th}}^{A} & \approx \sum_{i, j=4}^{5} \mathcal{S} \frac{\left|C_{A \tilde{\nu} \tilde{\nu}}\right|^{2}\left|C_{A \nu \nu}\right|^{2}}{16 m_{\chi}^{2} \Gamma_{A_{X}} T} \frac{\sqrt{1-c^{2}}}{c^{2}} \sqrt{1-\frac{4\left(\mu_{N S}\right)_{11}^{2}}{m_{A_{X}}^{2}}} \frac{K_{1}\left(\frac{m_{A_{X}}}{T}\right)}{K_{2}\left(\frac{m_{\tilde{\nu}_{1}^{R}}}{T}\right) K_{2}\left(\frac{m_{\tilde{\nu}}^{I}}{T}\right)} \\
& \approx \frac{\pi}{4} \frac{\tilde{C}_{A \tilde{\nu} \tilde{\nu}}^{2}}{2+\tilde{C}_{A \tilde{\nu} \tilde{\nu}}^{2}} \frac{1}{c^{3}} \sqrt{1-\frac{4\left(\mu_{N S}\right)_{11}^{2}}{m_{A_{X}}^{2}}} \frac{\lambda_{11}^{2}}{m_{\chi} T} \sqrt{\frac{c m_{\chi}}{\pi T}} \exp \left(-\frac{2(1-c) m_{\chi}}{c T}\right) \\
& \approx \frac{\pi}{2} \frac{\tilde{C}_{A \tilde{\nu} \tilde{\nu}}^{2}}{2+\tilde{C}_{A \tilde{\nu} \tilde{\nu}}^{2}} \frac{\sqrt{1-c^{2}}}{c^{3}} \frac{\lambda_{11}^{2}}{m_{\chi} T} \sqrt{\frac{c m_{\chi}}{\pi T}} \exp \left(-\frac{2(1-c) m_{\chi}}{c T}\right),
\end{aligned}
$$

where we have used the leading order term in the asymptotic expression of the modified Bessel function $K_{n}(x) \approx \sqrt{\pi / 2 x} \mathrm{e}^{-x}$ [48]. In the second line we have kept the factor with $\left(\mu_{N S}\right)_{11}^{2}$ as this makes it easier to estimate later how much the $H_{X}$-funnel is being more suppressed than this case.

By using the annihilation cross section formula eq. (4.23), we can numerically solve eq. (2) of ref. [49] to obtain the freeze-out point $x_{f}=m_{\chi} / T_{f}$ with the freeze-out temperature $T_{f}$ where we use the entropy table taken from ref. [50]. We then feed in the freeze-out temperature to calculate the relic density near the resonance as described in section $\mathrm{V}$ of ref. [49],

$$
\Omega_{\mathrm{DM}} h^{2}=\frac{1.079 \times 10^{9} \mathrm{GeV}^{-1}}{\sqrt{g_{*}} m_{\mathrm{pl}} J_{x_{f}}},
$$



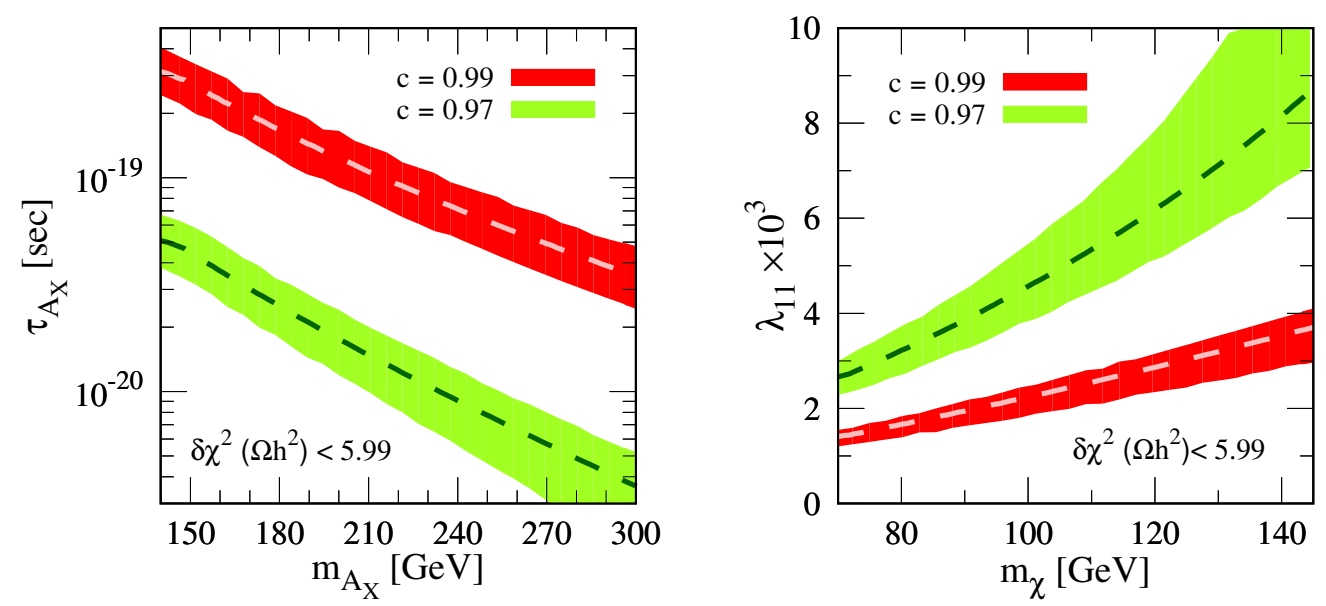

Figure 6. Results of our numerical scan where we have applied a $95 \%$ confidence level on the relic density. Dashed lines correspond to analytical result applied approximation of eq. (4.27) with $x_{f}=22$.

where

$$
\begin{aligned}
J_{x_{f}}= & \sum_{i, j=4}^{5} \mathcal{S}\left|C_{A \tilde{\nu} \tilde{\nu}}\right|^{2}\left|C_{A \nu \nu}\right|^{2} \frac{\left(m_{\tilde{\nu}_{1}^{R}}+m_{\tilde{\nu}_{1}^{I}}\right)^{2}}{4 m_{A_{X}}^{5} \Gamma_{A_{X}}} \sqrt{1-\frac{\left(\left|m_{\nu_{I}}\right|+\left|m_{\nu_{J}}\right|\right)^{2}}{m_{A_{X}}^{2}}} \\
& \times \operatorname{erfc}\left(\sqrt{x_{f}\left(1-\frac{\left(m_{\tilde{\nu}_{1}^{R}}+m_{\tilde{\nu}_{1}^{I}}\right)^{2}}{m_{A_{X}}^{2}}\right)}\right)(1+\Delta)^{3 / 2} \exp \left(-x_{f} \Delta\right)
\end{aligned}
$$

with $\Delta \equiv\left|m_{\tilde{\nu}_{1}^{R}}-m_{\tilde{\nu}_{1}^{I}}\right| / m_{\chi}$.

Again we can use some approximations to understand how the relic density scales approximately as

$$
J_{x_{f}}=\frac{\pi \lambda_{11}^{2}}{m_{\chi}^{2}} \frac{c^{4} \tilde{C}_{A \tilde{\nu} \tilde{\nu}}^{2}}{4\left(2+\tilde{C}_{A \tilde{\nu} \tilde{\nu}}^{2}\right)} \times \operatorname{erfc}\left(\sqrt{x_{f}\left(1-c^{2}\right)}\right) \exp \left(-x_{f} \Delta\right),
$$

where $\Delta$ can be rewritten by eq. (3.25) with input parameters as

$$
\Delta \approx\left|\frac{\lambda_{11} v_{X}}{4 m_{\chi}^{2}}\left(-\frac{8 m_{\chi}^{2}}{3 c^{2} \kappa_{0}\left(1-2.3 \kappa_{0}^{2}\right) v_{X}}-2 \sqrt{2} m_{\chi}+\kappa_{0} v_{X}\right)\right| .
$$

What we can clearly see from this approximation is that we expect an almost linear behavior of the relic density in the $\left(m_{\chi}, \lambda_{11}\right)$ plane. The complementary error function, erfc, is responsible for the strong dependence of the result on $c$. For $x_{f}=22$ and $c=0.99$ this piece evaluates to about 0.35 while for $x_{f}=22$ and $c=0.97$ it gives about 0.11 .

In figure 6 , we present the result of our numerical scan where we have applied a $95 \%$ confidence level, namely $\delta \chi^{2}\left(\Omega h^{2}\right)<5.99$, using the PLANCK result [51] and including conservative $10 \%$ theoretical uncertainties. The lifetime of $A_{X}$ is very short which justifies 
our assumption that the sneutrinos are in thermal equilibrium with the visible sector before freeze-out. In the $\left(m_{\chi}, \lambda_{11}\right)$ plane we see the anticipated approximate linear relation between $m_{\chi}$ and $\lambda_{11}$ although, in particular for $c=0.97$, there is a non-linear component which is coming mostly from the $\exp \left(-x_{f} \Delta\right)$ factor. The dependence of $\tilde{C}_{A \tilde{\nu} \tilde{\nu}}$ on $m_{\chi}$ is only subleading compared to that.

Of particular interest is that the allowed range for $\lambda$ corresponds very well to the ISS type III scenario discussed in [4].

\subsubsection{Scenario $H$ : sneutrino annihilation via the $H_{X}$-funnel}

In the model, there is another potential channel for the DM resonance, i.e. the CP-even boson which mostly contains the real component of the $X$ scalar. As depicted in the right panel of figure 5, the mediator and the species of the initial and final states are different from the $A_{X}$-funnel. Most notably this channel is a pure annihilation channel. What we will always implicitly assume is that $\tilde{\nu}_{1}^{R}$ is the DM candidate, which is the lighter sneutrino in the considered parameter range and we set $m_{\tilde{\nu}_{1}^{R}}=m_{\chi}$.

Analogous to the result in eq. (4.12) the annihilation cross section here reads

$$
\sigma(s)=\sum_{i, j=4}^{5} \frac{\left|C_{H \tilde{\nu} \tilde{\nu}}\right|^{2}\left|C_{H \nu \nu}\right|^{2}\left[s-\left(\left|m_{\nu_{i}}\right|+\left|m_{\nu_{j}}\right|\right)^{2}\right] \mathcal{S}}{8 \pi s\left[\left(s-m_{H_{X}}^{2}\right)^{2}+m_{H_{X}}^{2} \Gamma_{H_{X}}^{2}\right]} \sqrt{\frac{\left(s-m_{\nu_{j}}^{2}\right)^{2}-2 m_{\nu_{i}}^{2}\left(s+m_{\nu_{j}}^{2}\right)+m_{\nu_{i}}^{4}}{s\left(s-4 m_{\chi}^{2}\right)}}
$$

and $C_{H \tilde{\nu} \tilde{\nu}}$ and $C_{H \nu \nu}$ are $H_{X}$-sneutrino-sneutrino and $H_{X}-\nu_{i}-\nu_{j}$ couplings, respectively, and they are given as

$$
\begin{aligned}
C_{H \tilde{\nu} \tilde{\nu}}=\mathrm{i}\left(\kappa v_{X} \lambda_{11} Z_{16}^{R} Z_{16}^{I} Z_{23}^{H}+\frac{1}{\sqrt{2}}\left(A_{\lambda}\right)_{11} Z_{16}^{R} Z_{16}^{I} Z_{23}^{H}\right. \\
\left.\quad+\sqrt{2} \lambda_{11}\left(\mu_{N S}\right)_{11} Z_{14}^{R} Z_{16}^{I} Z_{23}^{H}+v_{X} \lambda_{11}^{2} Z_{16}^{R} Z_{16}^{I} Z_{23}^{H}\right) \\
\approx \mathrm{i} \frac{\lambda_{11}}{2} m_{H_{X}}\left(\frac{\left(\kappa+\lambda_{11}\right) v_{X}}{m_{H_{X}}}+\frac{1}{\sqrt{2}} \frac{A_{0}}{m_{H_{X}}}-\sqrt{2} \frac{\left(\mu_{N S}\right)_{11}}{m_{H_{X}}}\right), \\
C_{H \nu \nu}=-\frac{\mathrm{i}}{\sqrt{2}} \lambda_{11} U_{i 6}^{V} U_{j 6}^{V} Z_{23}^{H} \approx-\frac{\mathrm{i}}{2 \sqrt{2}} \lambda_{11} .
\end{aligned}
$$

The essential difference from the $A_{X}$-funnel is the factor with different mass dependence in the numerator due to the different coupling structure, i.e. here we have $s-\left(\left|m_{\nu_{i}}\right|+\left|m_{\nu_{j}}\right|\right)^{2}$ while in the $A_{X}$-funnel we had $s-\left(\left|m_{\nu_{i}}\right|-\left|m_{\nu_{j}}\right|\right)^{2}$.

The partial decay widths for $H_{X}$ are given by, similar to the $A_{X}$-funnel case:

$$
\begin{aligned}
\left.\Gamma_{H_{X}}\right|_{H_{X} \rightarrow 2 \nu_{4}} & =\frac{1}{32 \pi} \lambda_{11}^{2}\left(U_{46}^{V}\right)^{4} m_{H_{X}}\left(1-4\left(\frac{m_{\nu_{4}}}{m_{H_{X}}}\right)^{2}\right)^{3 / 2}, \\
\left.\Gamma_{H_{X}}\right|_{H_{X} \rightarrow 2 \nu_{5}} & =\left.\Gamma_{H_{X}}\right|_{H_{X} \rightarrow 2 \nu_{4}}(4 \rightarrow 5),
\end{aligned}
$$




$$
\begin{aligned}
\left.\Gamma_{H_{X}}\right|_{H_{X} \rightarrow \nu_{4} \nu_{5}} & =\frac{1}{16 \pi} \lambda_{11}^{2}\left(U_{46}^{V} U_{56}^{V}\right)^{2} m_{H_{X}} \sqrt{1-\frac{\left(m_{\nu_{5}}-m_{\nu_{4}}\right)^{2}}{m_{H_{X}}^{2}}}\left[1-\frac{\left(m_{\nu_{5}}+m_{\nu_{4}}\right)^{2}}{m_{H_{X}}^{2}}\right]^{3 / 2}, \\
\left.\Gamma_{H_{X}}\right|_{H_{X} \rightarrow \tilde{\nu}_{1}^{R} \tilde{\nu}_{1}^{I}} & =\frac{1}{16 \pi} \frac{\left|C_{H \tilde{\nu} \tilde{\nu}}\right|^{2}}{m_{H_{X}}} \sqrt{1-4\left(\frac{m_{\chi}}{m_{H_{X}}}\right)^{2}} .
\end{aligned}
$$

The thermal averaged cross section for the $H_{X}$-funnel is given analogous to the $A_{X}$-funnel as

$$
\begin{aligned}
\langle\sigma v\rangle_{\mathrm{th}}^{H}= & \sum_{i, j=4}^{5} \mathcal{S} \frac{\left|C_{H \tilde{\nu} \tilde{\nu}}\right|^{2}\left|C_{H \nu \nu}\right|^{2}\left[m_{H_{X}}^{2}-\left(m_{\nu_{i}}+m_{\nu_{j}}\right)^{2}\right]}{64 m_{\chi}^{4} \Gamma_{H_{X}} T} \sqrt{1-4\left(\frac{m_{\chi}}{m_{H_{X}}}\right)^{2}} \\
& \times \sqrt{\left(1-\frac{\left(m_{\nu_{i}}+m_{\nu_{j}}\right)^{2}}{m_{H_{X}}^{2}}\right)\left(1-\frac{\left(m_{\nu_{i}}-m_{\nu_{j}}\right)^{2}}{m_{H_{X}}^{2}}\right) \frac{K_{1}\left(\frac{m_{H_{X}}}{T}\right)}{\left[K_{2}\left(\frac{m_{\chi}}{T}\right)\right]^{2}} .}
\end{aligned}
$$

Similar to eq. (4.24), the thermal averaged cross section is simplified as

$$
\langle\sigma v\rangle_{\mathrm{th}}^{H} \approx \frac{\pi}{2} \frac{\tilde{C}_{H \tilde{\nu} \tilde{\nu}}^{2}}{2\left(1-c^{2}\right)+\tilde{C}_{H \tilde{\nu} \tilde{\nu}}^{2}} \frac{1}{c^{3}}\left(1-\frac{4\left(\mu_{N S}\right)_{11}^{2}}{m_{H_{X}}^{2}}\right)^{3 / 2} \frac{\lambda_{11}^{2}}{m_{\chi} T} \sqrt{\frac{c m_{\chi}}{\pi T}} \exp \left(-\frac{2(1-c) m_{\chi}}{c T}\right),
$$

where $\tilde{C}_{H \tilde{\nu} \tilde{\nu}}^{2}$ is

$$
\tilde{C}_{H \tilde{\nu} \tilde{\nu}}^{2} \equiv\left(\frac{\left(\kappa+\lambda_{11}\right) v_{X}}{m_{H_{X}}}+\frac{1}{\sqrt{2}} \frac{A_{0}}{m_{H_{X}}}-\sqrt{2} \frac{\left(\mu_{N S}\right)_{11}}{m_{H_{X}}}\right)^{2}
$$

which is also an $\mathcal{O}(1)$ factor. Comparing this approximation with eq. (4.24), we see that the thermal averaged cross section for the $H_{X}$-funnel has an extra suppression by

$$
1-4\left(\mu_{N S}\right)_{11}^{2} / m_{H_{X}}^{2} \sim 1-c^{2} \sim \mathcal{O}\left(10^{-2}\right)
$$

To compensate for this suppression, we need $\lambda_{11}$ to be larger by a factor of ten compared to the $A_{X}$-funnel, roughly speaking.

On the other hand, a larger $\lambda_{11}$ could lead to a critical problem of closing the annihilation channel into heavy neutrinos. If this channel was closed, only the annihilation into light neutrinos would be allowed, which is heavily suppressed and so the annihilation cross section would be too small by far. To avoid this we need

$$
s \approx m_{H_{X}}^{2}>\left(m_{\nu_{i}}+m_{\nu_{j}}\right)^{2} \approx 4\left(\mu_{N S}\right)_{11}^{2} \approx 4 m_{\chi}^{2}+\lambda_{11}\left(3 \kappa v_{X}^{2}-\frac{8}{\kappa c^{2}} m_{\chi}^{2}+2 \sqrt{2} m_{\chi} v_{X}\right),
$$

where we have used the results from section 3 and neglected terms of $\mathcal{O}\left(\lambda^{2}\right)$ and $\mathcal{O}\left(Y_{\nu}^{2}\right)$ and corrections to the masses of the heavy neutrinos. On the other hand $m_{\chi}^{2}=c^{2} / 4 m_{H_{X}}^{2}$ and in total

$$
\lambda_{11} \lesssim\left(\frac{4}{c^{2}}-4\right) m_{\chi}^{2}\left(3 \kappa v_{X}^{2}-\frac{8}{\kappa c^{2}} m_{\chi}^{2}+2 \sqrt{2} m_{\chi} v_{X}\right)^{-1}
$$



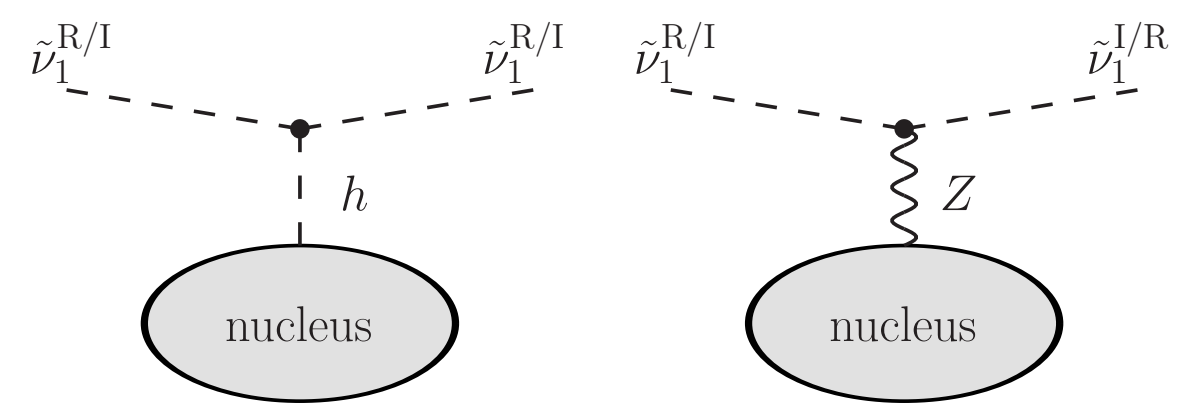

Figure 7. The relevant DM direct detection channels.

For a typical DM mass $m_{\chi}=100 \mathrm{GeV}$ with $c=0.97, \kappa=0.4$, and $\sqrt{2} v_{X}=1000 \mathrm{GeV}$ this means $\lambda_{11} \lesssim 4.3 \times 10^{-3}$ while from the comparison to the $A_{X}$-funnel we expect $\lambda_{11} \sim 2 \times 10^{-2}$. This rough estimate shows that the $H_{X}$-funnel is probably not working, which we have also confirmed numerically. Hence, we conclude that the $H_{X}$-funnel is not working within our setup.

\subsection{Dark matter direct and indirect detection}

In this section, we discuss direct and indirect detection prospects of our DM candidate. We begin with the direct detection which is difficult for discovery and then turn to indirect tests which are more promising but still far fetched.

\subsubsection{Dark matter direct detection}

The relevant diagrams for direct detection are shown in figure 7. There are two main channels connecting sneutrino DM to the nucleus. One is through the SM Higgs boson exchange and another is through the $Z$ boson exchange. The relevant effective Lagrangian for DM direct detection is

$$
\mathcal{L}_{\mathrm{DD}}^{\mathrm{Eff}} \simeq \frac{Y_{\nu}^{2} Y_{q} M_{\mathrm{SUSY}}}{m_{H}^{2}}\left(\tilde{\nu}_{1}^{R / I} \tilde{\nu}_{1}^{R / I}\right)(\bar{q} q)+\mathcal{C}_{Z}\left(\tilde{\nu}_{1}^{R} \tilde{\nu}_{1}^{I}\right)\left(\bar{q} \gamma_{5} q\right),
$$

where $M_{\text {SUSY }}$ is of order TeV. The contribution of the SM Higgs $H$ is larger than the other Higgs bosons due to either the much larger masses of the other MSSM Higgs bosons or the small Yukawa couplings to the quarks for $A_{X}$ and $H_{X}$. The effective coupling $\mathcal{C}_{Z}$ is

$$
\mathcal{C}_{Z}=\frac{e\left(1+\tan \theta_{W}\right) Y_{\nu}^{2} m_{q}\left(g_{L}^{(q)}-g_{R}^{(q)}\right)}{2 m_{Z}^{2}}
$$

with the weak mixing angle $\theta_{W}$, the quark mass $m_{q}$, and $\left(g_{L}^{(q)}-g_{R}^{(q)}\right)$ for up-type and down-type quarks is defined as

$$
\begin{aligned}
g_{L}^{(d)}-g_{R}^{(d)} & =\frac{e}{6}\left(\frac{3}{\tan \theta_{W}}-\tan \theta_{W}\right) \\
g_{L}^{(u)}-g_{R}^{(u)} & =-\frac{e}{2}\left(\frac{1}{\tan \theta_{W}}+\tan \theta_{W}\right) .
\end{aligned}
$$


We can see that the coefficients in front of the Higgs-exchange and the $Z$-exchange terms are roughly of the same order. On the other hand, the operators themselves give very different contributions to direct detection. Due to the presence of the $\gamma_{5}$ matrix in $\bar{q} \gamma_{5} q$, the contribution from the $Z$-exchange is highly suppressed. This is easily understood by considering the nonrelativistic expansion of the operator and studying its velocity dependence.

In the non-relativistic limit, the spinors involved in the scattering are $q=(\xi, \epsilon \xi)^{T}$ and $\bar{q}=\xi^{\dagger}(1, \epsilon) \gamma^{0}$, where $\xi$ is the two component Pauli spinor and $\epsilon=O(v / c)$. The velocity suppression for current DM in the Universe is $\epsilon \sim 10^{-4}-10^{-3}$. We can then do a simple expansion

$$
\begin{aligned}
\bar{q} q & =\left(\begin{array}{ll}
\xi^{\dagger} & \epsilon \xi^{\dagger}
\end{array}\right)\left(\begin{array}{cc}
1 & 0 \\
0 & -1
\end{array}\right)\left(\begin{array}{c}
\xi \\
\epsilon \xi
\end{array}\right)=\left(1-\epsilon^{2}\right) \xi^{\dagger} \xi \\
\bar{q} \gamma_{5} q & =\left(\begin{array}{ll}
\xi^{\dagger} & \epsilon \xi^{\dagger}
\end{array}\right)\left(\begin{array}{cc}
1 & 0 \\
0 & -1
\end{array}\right)\left(\begin{array}{ll}
0 & 1 \\
1 & 0
\end{array}\right)\left(\begin{array}{c}
\xi \\
\epsilon \xi
\end{array}\right)=O\left(\epsilon^{2}\right) \xi^{\dagger} \xi
\end{aligned}
$$

Therefore, we can easily see that the $Z$-exchange is suppressed by $O\left(\epsilon^{2}\right) \sim 10^{-8}-10^{-6}$ on amplitude level. We conclude that the $Z$-exchange is negligible compared to the Higgs exchange contribution and we focus on the Higgs exchange in the following.

The Higgs exchange cross section between DM and a nucleus is given by

$$
\sigma_{\tilde{\nu}_{1} N}(H)=\frac{1}{4 \pi} \frac{m_{N}^{2}}{\left(m_{\tilde{\nu}_{1}}+m_{N}\right)^{2}}\left[f_{p} Z+f_{n}(A-Z)\right]^{2} .
$$

The nucleus parameters, $A, Z$, and $m_{N}$ are the mass number, proton number, and the nucleus mass, respectively. The effective $\tilde{\nu}_{1}$-proton $\left(f_{p}\right)$ and $\tilde{\nu}_{1}$-neutron $\left(f_{n}\right)$ couplings for the Higgs channel are

$$
f_{p, n}^{i}=\frac{m_{p, n}}{v}\left[\sum_{q=u, d, s} f_{T_{q}} \frac{Y_{\nu}^{2} M_{\mathrm{SUSY}}}{m_{H}^{2}}+\sum_{Q=c, b, t} \frac{2}{27} f_{T_{G}} \frac{Y_{\nu}^{2} M_{\mathrm{SUSY}}}{m_{H}^{2}}\right],
$$

where $v=246 \mathrm{GeV}$. The numerical value of nucleon mass matrix elements $\left(f_{T_{q}}\right.$ and $\left.f_{T_{G}}\right)$ can be found in MicrOMEGAs [52].

Using $Y_{\nu}=10^{-6}$ and $M_{\mathrm{SUSY}}=1 \mathrm{TeV}$ we find a tiny DM-proton scattering cross section $\simeq 10^{-29} \mathrm{pb}$ (where $A=Z=1$ in eq. (4.46)) which is many orders of magnitude below the most stringent current limit $\sigma_{\tilde{\nu}_{1} p}^{\mathrm{SI}} \sim 5 \times 10^{-11} \mathrm{pb}$ for DM mass at $O(100) \mathrm{GeV}$ reported by the most recent XENON1T [53]. It is even below the neutrino floor which makes a direct detection rather difficult. On the other hand, a confirmed direct detection of DM in the near future would immediately rule out our setup, which is very attractive.

\subsubsection{Dark matter indirect detection}

Before we discuss indirect detection constraints we want to clarify that the DM is in fact a two-component dark matter. From eq. (3.25) the mass splitting between $\tilde{\nu}_{1}^{I}$ and $\tilde{\nu}_{1}^{R}$ is only $O(1) \mathrm{GeV}$. Therefore if $\tilde{\nu}_{1}^{I}$ is heavier it can decay only into $\tilde{\nu}_{1}^{R}$ and two active neutrinos via 
the $A_{X}$ boson. However, the coupling of $A_{X}$ to the active neutrinos is heavily suppressed and the phase space is tiny such that the lifetime of $\tilde{\nu}_{1}^{I}$ is estimated to be much longer than the present age of the Universe. The same argument is true if $\tilde{\nu}_{1}^{R}$ would be heavier. Thus, our model is an example of two component DM and since the sneutrinos are so close in mass we will assume for the sake of simplicity in this section that they homogeneously form $50 \%$ of DM each.

Since the DM candidate is strongly related to neutrinos, the most plausible idea is to look for a potential detection at IceCube [54] via monochromatically produced neutrinos from DM annihilation. The DM annihilation $\tilde{\nu}_{1}^{I} \tilde{\nu}_{1}^{R} \rightarrow \nu_{i} \nu_{j}$ via the $A_{X}$-funnel is the dominant annihilation channel at the Galactic center where the DM density is the highest in the Milky Way. Here, the indices $i$ and $j$ run from 1 to 5 . Note that sneutrino DM in this model can annihilate not only into heavy neutrinos but also into light active neutrinos but with a large suppression factor.

Since the mixing of the singlet fermion(s) with the active neutrinos is highly suppressed compared to those with heavy neutrinos, the most obvious channel to produce monochromatic active neutrinos is the annihilation into one active and one heavy neutrino, followed by subsequent decays of the heavy neutrino into active neutrinos. By the analogy from eq. (4.12), the annihilation cross section of the sneutrino DM into one active and one heavy neutrino can be written as,

$$
\sigma v_{\mathrm{rel}}\left(\tilde{\nu}_{1} \tilde{\nu}_{1} \rightarrow \nu_{\mathrm{active}} \nu_{K}\right)=\frac{C_{A \tilde{\nu} \tilde{\nu}}^{2}\left(C_{A \nu \nu}^{\prime}\right)^{2}}{4 \pi\left[\left(s-m_{A_{X}}^{2}\right)^{2}+m_{A_{X}}^{2} \Gamma_{A_{X}}^{2}\right]}\left(1-\frac{m_{\nu_{K}}^{2}}{s}\right)^{2},
$$

where $v_{\text {rel }} / 2=\sqrt{1-4 m_{\tilde{\nu}_{1}}^{2} / s}$, the $K=4,5$ represents the heavy neutrino and active neutrino masses are neglected. In addition, the coupling $C_{A \nu \nu}^{\prime}$ is similar to eq. (4.15)

$$
C_{A \nu \nu}^{\prime}=-\frac{1}{\sqrt{2}}\left(\lambda_{11} U_{i 6}^{V} U_{K 6}^{V}+\lambda_{22} U_{i 7}^{V} U_{K 7}^{V}\right) Z_{23}^{A},
$$

where $i$ runs from 1 to 3 . Since $U_{26}^{V} \lesssim \mathcal{O}\left(10^{-6}\right)$ and $U_{37}^{V} \lesssim \mathcal{O}\left(10^{-6}\right)$ we obtain $\left(C_{A \nu \nu}^{\prime}\right)^{2} \simeq$ $\mathcal{O}\left(10^{-18}\right)$ after combining with typical values of $\lambda_{11}$. Note that due to tiny mixings, the suppression factor for direct annihilation into two active neutrinos is $\sim \mathcal{O}\left(10^{-12}\right)$ compared to the annihilation into one active and one heavy neutrino.

For the DM annihilation into one active and one heavy neutrino we hence estimate an annihilation cross section of $\mathcal{O}\left(10^{-41}\right) \mathrm{cm}^{3} \mathrm{~s}^{-1}$ in our model. IceCube sets the most stringent limit on DM annihilation cross sections into monochromatic neutrino lines of around $2 \times 10^{-23} \mathrm{~cm}^{3} \mathrm{~s}^{-1}$ at $m_{\chi} \sim \mathcal{O}(100) \mathrm{GeV}$ [54] using the Navarro-Frenk-White DM profile, which is much larger than our prediction and a search for monochromatic neutrino lines from our DM annihilation is not very promising. However, there might be some chance to probe the small DM annihilation cross section well below $\mathcal{O}\left(10^{-26}\right) \mathrm{cm}^{3} \mathrm{~s}^{-1}$ by looking for the neutrino line signature boosted by over densities of dark matter spiked around a supermassive or an intermediate black hole hosted in spheroidal galaxies [55]. Naive extrapolation indicates that the quantity energy neutrino times neutrino flux $E \Phi_{\nu_{\text {line }}}$ is several orders of magnitude below the future sensitivity of IceCube-Gen2. 


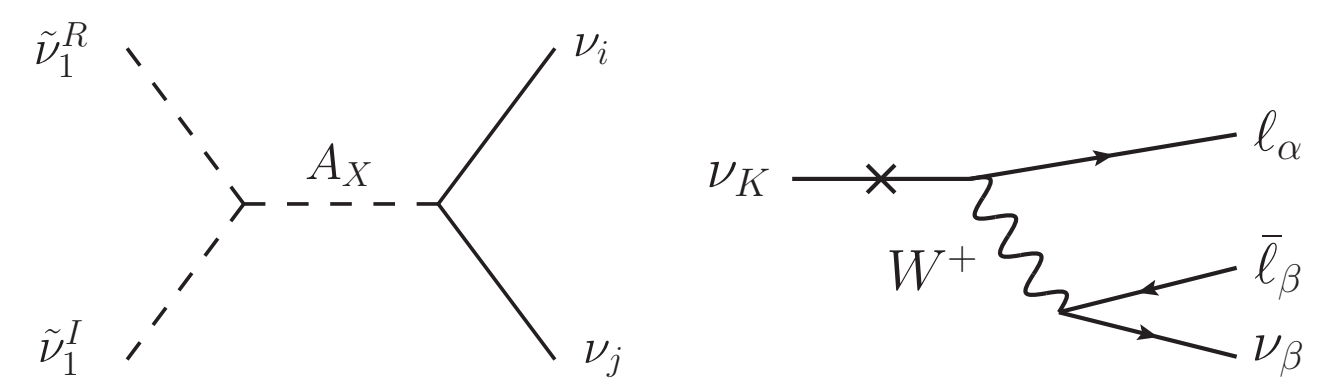

Figure 8. (Left) Annihilation channel for DM into a pair of neutrinos, where $i, j$ runs from 1 to 5. When $i=1-3$ and $j=K=4,5$ corresponds to annihilation into one active and one heavy neutrinos. (Right) The dominant decay of the heavy neutrino into an active neutrino and charged leptons. Here $K$ is 4 or 5 .

Nevertheless, the story simply does not end here if we consider the heavy neutrino cascade decays into SM particles, such as electrons and positrons, and also the bremsstrahlung photons or inverse-compton-scattered photons. Typical cascade decays of heavy neutrinos into leptons are depicted in the right panel of figure 8. For DM annihilation into heavy neutrinos only and subsequent decays into leptons, one can take the coupling $C_{A \nu \nu}^{\prime} \sim \lambda_{11} / 2 \sqrt{2}$, cf. eq. (4.15). We found that the annihilation cross section $\left\langle\sigma v_{\text {rel }}\right\rangle$ of the process shown in figure 8 can be as large as $\mathcal{O}\left(10^{-29}\right) \mathrm{cm}^{3} \mathrm{~s}^{-1}$. This prediction is only a few orders of magnitude below the current limit of $10^{-26} \mathrm{~cm}^{3} \mathrm{~s}^{-1}$ from leptophilic DM channels of the AMS-02 [56]. Hence, there may be some chance that such charged leptons and secondary photons can be probed in future cosmic-ray experiments.

In summary, predictions of our model in both direct and indirect detection are very safe with the most stringent current limits. Inversely speaking, once DM direct or indirect detection finds any DM signal, our model can be easily excluded by observations.

\section{$5 \quad$ Summary and conclusions}

Neutrino masses and dark matter are two of the most established evidences for physics beyond the SM. In a previous work we have proposed a supersymmetric inverse seesaw model that adds neutrino masses to the MSSM. The seesaw scale of that model is of the electroweak scale since it is determined by SUSY breaking parameters. To be more precise the vev of the scalar component of $\hat{X}$ induces the lepton number violating terms in the neutrino mass matrix and the smallness of neutrino masses is then given by some smallish Yukawa couplings and we can reproduce neutrino oscillation data perfectly.

In this work we have focused on the novel dark matter aspects of this model compared to the MSSM. First of all, the stability of the LSP is guaranteed because of the unbroken DM parity embedded in our model which acts like matter parity on the MSSM fields and hence forbids all $R$-parity violating operators. The sneutrinos which are odd under this parity thus form a potential dark matter candidate. In this work the lightest sneutrinos which are mainly a linear combination of the scalar components of the right-handed neutrino $\hat{N}^{c}$ and singlet $\hat{S}$ superfields are thermally produced DM particles. 
We have shown that the co-annihilation rate of the CP-even and the CP-odd sneutrinos can be sizable around the resonance peak of the pseudoscalar boson $A_{X}$, the $A_{X}$-funnel, and gives the right amount of DM relic density of the Universe. The $A_{X}$ is the pseudoscalar component of the $\hat{X}$ superfield which plays the crucial role to generate the light neutrino masses as mentioned above. Hence, in our model we have a very close relationship between the neutrino and the DM sector. Note also that the current DM population is a mix of $\mathrm{CP}$-even and CP-odd sneutrinos in our setup.

Furthermore, we have estimated the scattering rate of the sneutrino DM with nuclei, and found that the dominant contribution comes from the Higgs-boson exchange. Yet, it is many orders of magnitude below the current DM direct detection limits. Thus, any signals in direct-detection experiments can immediately rule out our setup. Of course, in that case we could try to embed, for instance, conventional neutralino DM if in agreement with data. Similarly, the monochromatic neutrino line signal is a smoking-gun signature of the model, but the rate is way below the current IceCube limit. Nevertheless, the indirect detection may stand a chance to observe the annihilation of the dark matter particles into heavy neutrinos followed by their subsequent cascade decays into charged leptons and photons in cosmic-ray and gamma-ray telescopes.

Let us again briefly highlight a few important findings of this work before we conclude:

1. There is a tiny mass splitting between the real and imaginary components of the sneutrino DM. The annihilation via the $A_{X}$-funnel, which is a pseudoscalar, has to involve the real and imaginary parts and is in fact a co-annihilation.

2. The mass of $A_{X}$ is about two times of the sneutrino DM, such that the co-annihilation rate can be sufficiently enhanced so as not to overclose the Universe.

3. In principal, there is the $H_{X}$-funnel as well but it turns out that the annihilation rate in this channel is much smaller than in the $A_{X}$-funnel due to $p$-wave suppression and too small to get the right relic density.

4. The scattering cross section of the sneutrino DM with nuclei is extremely small because of the tiny Yukawa couplings with the ordinary Higgs boson such that it is well below existing limits.

5. There may be some chance to observe the charged leptons or secondary photons coming from the co-annihilation of the sneutrino DM in the galactic halo.

6. The most striking feature of our DM is the close link to the neutrino sector through its couplings to the scalar $X$-bosons. This is reflected in its phenomenology as well since DM co-annihilates exclusively into neutrinos.

7. The fermionic component of the $\hat{X}$ superfield, the Xino, could also be the LSP. However, its dominant annihilation into $A_{X} H_{X}$ in general has a too large cross section because of the $\mathcal{O}(1)$ coupling $\kappa$ to give a significant contribution to the relic density. Only when the phase space for this process closes the annihilation cross section could be sufficiently suppressed. 
In this paper we have worked out another striking feature of our supersymmetric electroweak scale inverse seesaw model, namely the possible close connection between dark matter and neutrinos. Hence, apart from the rather technical hierarchy problem we can solve two of the most outstanding experimental challenges to the SM, neutrino masses and dark matter. In the future, we will explore other features of our model related to collider physics and leptogenesis with hopefully similarly interesting findings.

\section{Acknowledgments}

We would like to thank Florian Staub for helping us to implement our model into SARAH properly. This research was supported in parts by the Ministry of Science and Technology (MoST) of Taiwan under Grant No. MOST-105-2112-M-007-028-MY3. J.C. was supported by the National Research Foundation of Korea (NRF) grant No. NRF2016R1E1A1A01943297.

Open Access. This article is distributed under the terms of the Creative Commons Attribution License (CC-BY 4.0), which permits any use, distribution and reproduction in any medium, provided the original author(s) and source are credited.

\section{References}

[1] G. Bertone, D. Hooper and J. Silk, Particle dark matter: Evidence, candidates and constraints, Phys. Rept. 405 (2005) 279 [hep-ph/0404175] [INSPIRE].

[2] Super-Kamiokande collaboration, Y. Fukuda et al., Evidence for oscillation of atmospheric neutrinos, Phys. Rev. Lett. 81 (1998) 1562 [hep-ex/9807003] [INSPIRE].

[3] SNO collaboration, Q.R. Ahmad et al., Direct evidence for neutrino flavor transformation from neutral current interactions in the Sudbury Neutrino Observatory, Phys. Rev. Lett. 89 (2002) 011301 [nucl-ex/0204008] [INSPIRE].

[4] J. Chang, K. Cheung, H. Ishida, C.-T. Lu, M. Spinrath and Y.-L.S. Tsai, A supersymmetric electroweak scale seesaw model, JHEP 10 (2017) 039 [arXiv:1707.04374] [INSPIRE].

[5] J.S. Hagelin, G.L. Kane and S. Raby, Perhaps Scalar Neutrinos Are the Lightest Supersymmetric Partners, Nucl. Phys. B 241 (1984) 638 [InSPIRE].

[6] L.E. Ibáñez, The Scalar Neutrinos as the Lightest Supersymmetric Particles and Cosmology, Phys. Lett. B 137 (1984) 160 [INSPIRE].

[7] T. Falk, K.A. Olive and M. Srednicki, Heavy sneutrinos as dark matter, Phys. Lett. B 339 (1994) 248 [hep-ph/9409270] [INSPIRE].

[8] N. Arkani-Hamed, L.J. Hall, H. Murayama, D. Tucker-Smith and N. Weiner, Small neutrino masses from supersymmetry breaking, Phys. Rev. D 64 (2001) 115011 [hep-ph/0006312] [INSPIRE].

[9] D. Hooper, J. March-Russell and S.M. West, Asymmetric sneutrino dark matter and the Omega(b)/Omega(DM) puzzle, Phys. Lett. B 605 (2005) 228 [hep-ph/0410114] [INSPIRE].

[10] C. Arina and N. Fornengo, Sneutrino cold dark matter, a new analysis: Relic abundance and detection rates, JHEP 11 (2007) 029 [arXiv:0709.4477] [INSPIRE]. 
[11] C. Arina, F. Bazzocchi, N. Fornengo, J.C. Romao and J.W.F. Valle, Minimal supergravity sneutrino dark matter and inverse seesaw neutrino masses, Phys. Rev. Lett. 101 (2008) 161802 [arXiv: 0806.3225] [INSPIRE].

[12] K.-Y. Choi and O. Seto, Light Dirac right-handed sneutrino dark matter, Phys. Rev. D 88 (2013) 035005 [arXiv: 1305.4322] [INSPIRE].

[13] T. Asaka, K. Ishiwata and T. Moroi, Right-handed sneutrino as cold dark matter, Phys. Rev. D 73 (2006) 051301 [hep-ph/0512118] [INSPIRE].

[14] T. Asaka, K. Ishiwata and T. Moroi, Right-handed sneutrino as cold dark matter of the universe, Phys. Rev. D 75 (2007) 065001 [hep-ph/0612211] [INSPIRE].

[15] J. McDonald, Right-handed sneutrino condensate cold dark matter and the baryon-to-dark matter ratio, JCAP 01 (2007) 001 [hep-ph/0609126] [INSPIRE].

[16] V. Page, Non-thermal right-handed sneutrino dark matter and the $\Omega_{\mathrm{DM}} / \Omega_{b}$ problem, JHEP 04 (2007) 021 [hep-ph/0701266] [INSPIRE].

[17] H.-S. Lee, K.T. Matchev and S. Nasri, Revival of the thermal sneutrino dark matter, Phys. Rev. D 76 (2007) 041302 [hep-ph/0702223] [INSPIRE].

[18] D.G. Cerdeno, C. Muñoz and O. Seto, Right-handed sneutrino as thermal dark matter, Phys. Rev. D 79 (2009) 023510 [arXiv: 0807. 3029] [INSPIRE].

[19] L. Delle Rose, S. Khalil, S.J.D. King, C. Marzo, S. Moretti and C.S. Un, Naturalness and dark matter in the supersymmetric B-L extension of the standard model, Phys. Rev. D 96 (2017) 055004 [arXiv: 1702.01808] [INSPIRE].

[20] L. Delle Rose et al., Sneutrino Dark Matter in the BLSSM, JHEP 07 (2018) 100 [arXiv: 1712.05232] [INSPIRE].

[21] L. Delle Rose et al., Prospects for Sneutrino Dark Matter in the BLSSM, arXiv:1804.09470 [INSPIRE].

[22] G.R. Farrar and P. Fayet, Phenomenology of the Production, Decay and Detection of New Hadronic States Associated with Supersymmetry, Phys. Lett. B 76 (1978) 575 [INSPIRE].

[23] S. Dimopoulos and H. Georgi, Softly Broken Supersymmetry and SU(5), Nucl. Phys. B 193 (1981) 150 [INSPIRE].

[24] S. Weinberg, Supersymmetry at Ordinary Energies. 1. Masses and Conservation Laws, Phys. Rev. D 26 (1982) 287 [INSPIRE].

[25] N. Sakai and T. Yanagida, Proton Decay in a Class of Supersymmetric Grand Unified Models, Nucl. Phys. B 197 (1982) 533 [INSPIRE].

[26] S. Dimopoulos, S. Raby and F. Wilczek, Proton Decay in Supersymmetric Models, Phys. Lett. B 112 (1982) 133 [INSPIRE].

[27] P.S. Bhupal Dev, S. Mondal, B. Mukhopadhyaya and S. Roy, Phenomenology of Light Sneutrino Dark Matter in cMSSM/mSUGRA with Inverse Seesaw, JHEP 09 (2012) 110 [arXiv: 1207.6542] [INSPIRE].

[28] S. Banerjee, P.S.B. Dev, S. Mondal, B. Mukhopadhyaya and S. Roy, Invisible Higgs Decay in a Supersymmetric Inverse Seesaw Model with Light Sneutrino Dark Matter, JHEP 10 (2013) 221 [arXiv: 1306.2143] [INSPIRE]. 
[29] J. Guo, Z. Kang, T. Li and Y. Liu, Higgs boson mass and complex sneutrino dark matter in the supersymmetric inverse seesaw models, JHEP 02 (2014) 080 [arXiv:1311.3497] [INSPIRE].

[30] D.K. Ghosh, S. Mondal and I. Saha, Confronting the Galactic Center Gamma Ray Excess With a Light Scalar Dark Matter, JCAP 02 (2015) 035 [arXiv:1405.0206] [INSPIRE].

[31] J. Cao, X. Guo, Y. He, L. Shang and Y. Yue, Sneutrino DM in the NMSSM with inverse seesaw mechanism, JHEP 10 (2017) 044 [arXiv: 1707.09626] [INSPIRE].

[32] I. Gogoladze, B. He, A. Mustafayev, S. Raza and Q. Shafi, Effects of Neutrino Inverse Seesaw Mechanism on the Sparticle Spectrum in CMSSM and NUHM2, JHEP 05 (2014) 078 [arXiv: 1401.8251] [INSPIRE].

[33] Z. Kang, J. Li, T. Li, T. Liu and J.M. Yang, The maximal U(1) $)_{L}$ inverse seesaw from $d=5$ operator and oscillating asymmetric Sneutrino dark matter, Eur. Phys. J. C 76 (2016) 270 [arXiv: 1102.5644] [INSPIRE].

[34] S.-L. Chen and Z. Kang, Oscillating asymmetric sneutrino dark matter from the maximally $\mathrm{U}(1)_{L}$ supersymmetric inverse seesaw, Phys. Lett. B 761 (2016) 296 [arXiv:1512.08780] [INSPIRE].

[35] S. Khalil, H. Okada and T. Toma, Right-handed Sneutrino Dark Matter in Supersymmetric B-L Model, JHEP 07 (2011) 026 [arXiv:1102.4249] [INSPIRE].

[36] H. An, P.S.B. Dev, Y. Cai and R.N. Mohapatra, Sneutrino Dark Matter in Gauged Inverse Seesaw Models for Neutrinos, Phys. Rev. Lett. 108 (2012) 081806 [arXiv:1110.1366] [INSPIRE].

[37] D. Borah, Astrophysical Constraints on the scale of Left-Right Symmetry in Inverse Seesaw Models, J. Mod. Phys. 3 (2012) 1097 [arXiv: 1204.6587] [InSPIRE].

[38] W. Abdallah and S. Khalil, Dark Matter in B-L supersymmetric Standard Model with inverse seesaw, JCAP 04 (2017) 016 [arXiv: 1701.04436] [INSPIRE].

[39] V. De Romeri and M. Hirsch, Sneutrino Dark Matter in Low-scale Seesaw Scenarios, JHEP 12 (2012) 106 [arXiv:1209.3891] [INSPIRE].

[40] M. Frank and Ö. Özdal, Exploring the supersymmetric $\mathrm{U}(1)_{B-L} \times \mathrm{U}(1)_{R}$ model with dark matter, muon $g-2$ and $Z^{\prime}$ mass limits, Phys. Rev. D 97 (2018) 015012 [arXiv:1709.04012] [INSPIRE].

[41] F. Staub, Sarah, arXiv:0806.0538 [INSPIRE].

[42] F. Staub, SARAH 4: A tool for (not only SUSY) model builders, Comput. Phys. Commun. 185 (2014) 1773 [arXiv:1309.7223] [INSPIRE].

[43] A. Fowlie et al., The CMSSM Favoring New Territories: The Impact of New LHC Limits and a 125 GeV Higgs, Phys. Rev. D 86 (2012) 075010 [arXiv: 1206.0264] [INSPIRE].

[44] I. Esteban, M.C. Gonzalez-Garcia, M. Maltoni, I. Martinez-Soler and T. Schwetz, Updated fit to three neutrino mixing: exploring the accelerator-reactor complementarity, JHEP 01 (2017) 087 [arXiv: 1611.01514] [INSPIRE].

[45] W. Porod, SPheno, a program for calculating supersymmetric spectra, SUSY particle decays and SUSY particle production at $e^{+} e^{-}$colliders, Comput. Phys. Commun. 153 (2003) 275 [hep-ph/0301101] [INSPIRE]. 
[46] W. Porod and F. Staub, SPheno 3.1: Extensions including flavour, CP-phases and models beyond the MSSM, Comput. Phys. Commun. 183 (2012) 2458 [arXiv:1104.1573] [inSPIRE].

[47] J. Edsjö and P. Gondolo, Neutralino relic density including coannihilations, Phys. Rev. D 56 (1997) 1879 [hep-ph/9704361] [INSPIRE].

[48] M. Srednicki, R. Watkins and K.A. Olive, Calculations of Relic Densities in the Early Universe, Nucl. Phys. B 310 (1988) 693 [InSPIRE].

[49] K. Griest and D. Seckel, Three exceptions in the calculation of relic abundances, Phys. Rev. D 43 (1991) 3191 [INSPIRE].

[50] M. Drees, F. Hajkarim and E.R. Schmitz, The Effects of QCD Equation of State on the Relic Density of WIMP Dark Matter, JCAP 06 (2015) 025 [arXiv:1503.03513] [INSPIRE].

[51] Planck collaboration, P.A.R. Ade et al., Planck 2015 results. XIII. Cosmological parameters, Astron. Astrophys. 594 (2016) A13 [arXiv:1502.01589] [INSPIRE].

[52] G. Bélanger, F. Boudjema, A. Pukhov and A. Semenov, MicrOMEGAs 2.0: A program to calculate the relic density of dark matter in a generic model, Comput. Phys. Commun. 176 (2007) 367 [hep-ph/0607059] [INSPIRE].

[53] XENON collaboration, E. Aprile et al., Dark Matter Search Results from a One Tonne Year Exposure of XENON1T, arXiv: 1805.12562 [INSPIRE].

[54] IcECube collaboration, M.G. Aartsen et al., Search for Neutrinos from Dark Matter Self-Annihilations in the center of the Milky Way with 3 years of IceCube/DeepCore, Eur. Phys. J. C 77 (2017) 627 [arXiv: 1705.08103] [INSPIRE].

[55] C. Arina, S. Kulkarni and J. Silk, Monochromatic neutrino lines from sneutrino dark matter, Phys. Rev. D 92 (2015) 083519 [arXiv:1506.08202] [INSPIRE].

[56] L.A. Cavasonza, H. Gast, M. Krämer, M. Pellen and S. Schael, Constraints on leptophilic dark matter from the AMS-02 experiment, Astrophys. J. 839 (2017) 36 [arXiv:1612.06634] [INSPIRE]. 